УДК 550.83

DOI: $10.23671 /$ VNC.2016.3.20831

\title{
ВЫЧИСЛЕНИЕ ВЫСШИХ ПРОИЗВОДНЫХ ГРАВИТАЦИОННОГО ПОТЕНЦИАЛА НА ОСНОВЕ F-АППРОКСИМАЦИИ
}

\author{
() 2016 И.А. Керимов, д.ф.-м.н., профр. \\ Институт фризики Земли им. О.Ю. Шмидта РАН, Россия, 123995, г. Москва, ул. \\ Б. Грузинская, 10, стр. 1, e-mail: kerimov@ ifz.ru
}

В статье рассмотрен метод транссрормации гравитационного поля (определение различных компонент потенциала силы тяжести) на аппроксимационной основе. Разработанный автором метод и компьютерные технологии F-аппроксимации позволяют вычислять горизонтальные и вертикальные производные гравитационного потенциала для исходных данных, заданных как на регулярной, так и на нерегулярной сети. Метод апробирован на модельных и фактических гравиметрических данных. мация.

Ключевые слова: аппроксимация, высшие производные, аномалия, гравитационное поле, трансфор-

Трансформации аномалий силы тяжести (нахождение пространственного распределения поля и его производных, разделение полей, осреднение, сглаживание, пересчет их в некоторые другие функции и др.) достаточно широко применяются в практике обработки и интерпретации гравитационных данных. Как известно, гравитационному потенциалу и его производным присуще так называемое свойство аддитивности, которое заключается в том, что значение функции от суммы аргументов равняется сумме значений функции от каждого аргумента в отдельности [Гравиразведка, 1990; Керимов, 2015; Керимов и др., 2008; Маловичко, Тарунина, 1981; Страхов, Токарь, 1985; Тарунина, 1995].

Как известно, трансформация гравитационных аномалий выполняется на основе априорных предпосылок (диапазон глубин, в котором заключены источники аномалий, либо минимальные и максимальные значениями градиента поля и др.). Одним из наиболее эффективных способов трансформации является вычисление высшие производные потенциала силы тяжести [Гравиразведка, 1990; Керимов, 2011б; Маловичко, Тарунина, 1981; Страхов, Токарь, 1985; Тарунина, 1995]. Большинство известных методов вычисления высших производные потенциала являются неустойчивыми. Недостатком большинства существующих методов является также их неадекватность реальной геофизической практике (не учет нерегулярности и разновысотности гравиметрических сетей и другие идеализации) [Керимов, 2011a, 2015; Керимов и др., 2008; Маловичко, Тарунина, 1981].

Использование аппроксимационного подхода к спектральному анализу, основанного на методе линейных интегральных представлений В.Н. Страхова, позволяет устранить недостатки существующих методов [Керимов, 2011a, б, 2003, 2009а, б, 2015; Керимов и др., 2008; Маловичко, Тарунина, 1981; Страхов, 1992, 1999, 2001; Страхов и др., 2009]. Теоретические основы, алгоритмы и компьютерные программы аппроксимационного подхода к спектральному анализу, получившего название метод F-аппроксимации, описаны в ряде работ [Керимов, 2011a, б, 2003, 2009а, б, 2015; Страхов, 1999, 2001]. Результаты апробирования метода на модельных и фактических геофизических данных, заданных на нерегулярной сети, позволили 
сделать вывод о высокой точности восстановления поля путем F-аппроксимации [Керимов, 2011a, 2009a, б; Страхов, 1999]. Метод F-аппроксимации позволяет также принципиально по-новому решать ряд вопросов трансформаций потенциальных полей.

В данной статье в продолжение предыдущих работ автора по нахождению линейных трансформаций потенциальных полей рассмотрены алгоритмы и компьютерные технологии пространственного распределения поля и его производных на основе F-аппроксимации. Приводятся также результаты опробования алгоритмов и компьютерных технологий на модельных и практических геолого-гравиметрических материалах.

В предыдущих работах [Керимов, 2011а, б, 2003, 2009а, б; Страхов, 1999] было получено выражение для первой вертикальной производной гравитационного потенциала:

где

$$
W_{z}\left(\xi_{l, l}, \xi_{2, l}, \xi_{3, l}\right)=\frac{3}{2 \pi} \sum_{k=1}^{N} \lambda_{k} \frac{\left(2 z_{k, l}^{2}-3 p_{k, l}^{2}\right) \cdot z}{\left(z_{k, l}^{2}+p_{k, l}^{2}\right)^{\frac{7}{2}}},
$$

$$
\begin{gathered}
p_{k, l}=\sqrt{\left(x_{1, k}-\xi_{1, l}\right)^{2}+\left(x_{2, k}-\xi_{2, l}\right)^{2}} \\
z_{k, l}=x_{3, k}+\xi_{3, l}+2 H
\end{gathered}
$$

С учетом (1) и (2) окончательно имеем формулы для расчета различных элементов гравитационного поля на заданном уровне $\mathrm{h}(\mathrm{h}<\mathrm{H})$. Полученные формулы (4-9) могут быть использованы для пересчета поля силы тяжести и его производных на заданный уровень $h$ как на нерегулярной, так и на регулярной сети. С целью оценки точности аналитического продолжения гравитационного поля на основе F-аппроксимации были выполнены расчеты на модельных участках №№ 1-3. Используемые модели описаны в работах [Керимов, 2003, 2011a, б, 2015; Страхов и др., 2009]. Результаты расчетов приведены в таблицах №№ 1-3.

Для оценки относительной точности аппроксимации использовалась следующая мера:

где:

$$
\gamma_{k}=\frac{\left\|f_{u c m}-f_{\text {Bbu }}^{(k)}\right\|_{E}}{\left\|f_{u c m}\right\|_{E}}
$$

$f_{u c m}-$ исходные (в случае модельных примеров точное) значения гравитационного поля;

$f_{\text {выч }}^{(k)}$ - восстановленные по результатам аппроксимации значения поля при решении СЛАУ различными методами.

Для оценки среднеквадратических погрешностей аппроксимации, полученных при решении СЛАУ использовалась следующая формула:

$$
\sigma_{k}=\frac{\left\|f_{\text {bblu }}^{(k)}-f_{u c m}\right\|_{E}}{\sqrt{N}}
$$

Рассмотрим особенности решения некоторых задач (восстановления поля, измеренного на нерегулярной сети, на регулярной сети, пересчет наблюденного поля на заданный уровень, расчет горизонтальных и вертикальных производных на различных уровнях и др.). 


\section{Вычисление гравитационного потенциала $W(\xi)$ и его производных}

Учетом вышеизложенного выражение для гравитационного потенциала имеет следующий вид:

$$
W\left(\xi_{1, l}, \xi_{2, l}, \xi_{3, l}\right)=\frac{1}{2 \pi} \sum_{k=1}^{N} \lambda_{k} \frac{2 z_{k, l}^{2}-p_{k, l}^{2}}{\left(z_{k, l}^{2}+p_{k, l}^{2}\right)^{3.5}},
$$

где $p_{k, l}$ и $z_{k, l}$ определены в соответствии с формулами (2) и (3).

Соответственно выражение для первой вертикальной производной гравитационного потенциала:

$$
W_{z}\left(\xi_{l, l}, \xi_{2, l}, \xi_{3, l}\right)=\frac{3}{2 \pi} \sum_{k=1}^{N} \lambda_{k} \frac{\left(2 z_{k, l}^{2}-3 p_{k, l}^{2}\right) \cdot z}{\left(z_{k, l}^{2}+p_{k, l}^{2}\right)^{3.5}}
$$

Полученная формула (7) для вычисления первой вертикальной производной может быть использована для восстановления поля силы тяжести на нерегулярной или регулярной сети, а также для пересчета на заданный уровень. Аналогичные выражения можно получить для первых горизонтальных производных гравитационного потенциала:

$$
\begin{aligned}
& W_{x}\left(\xi_{l, l}, \xi_{2, l}, \xi_{3, l}\right)=\frac{3}{2 \pi} \sum_{k=1}^{N} \lambda_{k} \frac{\left(x_{l, k}-\xi_{l, l}\right)\left(p_{k, l}^{2}-4 z_{k, l}^{2}\right)}{\left(z_{k, l}^{2}+p_{k, l}^{2}\right)^{3.5}} \\
& W_{y}\left(\xi_{l, l}, \xi_{2, l}, \xi_{3, l}\right)=\frac{3}{2 \pi} \sum_{k=1}^{N} \lambda_{k} \frac{\left(x_{2, k}-\xi_{2, l}\right)\left(p_{k, l}^{2}-4 z_{k, l}^{2}\right)}{\left(z_{k, l}^{2}+p_{k, l}^{2}\right)^{3.5}}
\end{aligned}
$$

Аналогичный рассмотренному выше подход может быть использован для получения выражений для вторых горизонтальных и вертикальной производной гравитационного потенциала. В свою очередь на основании выражений для вторых горизонтальных производных можно получить формулы для определения модуля и направления полного горизонтального градиента силы тяжести. Окончательные выражения для вторых горизонтальных и вертикальной производных гравитационного потенциала имеют следующий вид:

$$
\begin{array}{r}
W_{x z}\left(\xi_{l, l}, \xi_{2, l}, \xi_{3, l}\right)=\frac{15}{\pi} \sum_{k=1}^{N} \lambda_{k} \frac{\left(x_{1, k}-\xi_{1, l}\right)\left(3 \rho_{k, l}^{2}-4 z_{k, l}^{3}\right)}{\left(z_{k, l}^{2}+\rho_{k, l}^{2}\right)^{4.5}} \\
W_{y z}\left(\xi_{1, l}, \xi_{2, l}, \xi_{3, l}\right)=\frac{15}{\pi} \sum_{k=1}^{N} \lambda_{k} \frac{\left(x_{2, k}-\xi_{2, l}\right)\left(3 \rho_{k, l}^{2}-4 z_{k, l}^{3}\right)}{\left(z_{k, l}^{2}+\rho_{k, l}^{2}\right)^{4.5}} \\
W_{s z}\left(\xi_{1, l}, \xi_{2, l}, \xi_{3, l}\right)=\frac{15}{\pi} \sum_{k=1}^{N} \lambda_{k} \frac{\left(3 \rho_{k, l}^{2}-4 z_{k, l}^{3}\right) p_{k, l}}{\left(z_{k, l}^{2}+\rho_{k, l}^{2}\right)^{4.5}} \\
W_{z z}\left(\xi_{1, l}, \xi_{2, l}, \xi_{3, l}\right)=\frac{15}{\pi} \sum_{k=1}^{N} \lambda_{k} \frac{8 z_{k, l}^{4}-24 \rho_{k, l}^{2} z_{k, l}^{2}+3 \rho_{k, l}^{4}}{\left(z_{k, l}^{2}+\rho_{k, l}^{2}\right)^{4.5}}
\end{array}
$$

После очевидных преобразований получим формулы для вычисления третьих производных гравитационного потенциала: 


$$
\begin{gathered}
W_{z z z}\left(\xi_{1, l}, \xi_{2, l}, \xi_{3, l}\right)=\frac{75}{\pi} \sum_{k=1}^{N} \lambda_{k} \frac{z_{k, l}\left(8 z_{k, l}^{4}-40 \rho_{k, l}^{2} z_{k, l}^{2}+15 \rho_{k, l}^{4}\right)}{\left(z_{k, l}^{2}+\rho_{k, l}^{2}\right)^{5.5}} \\
W_{x x z}\left(\xi_{l, l}, \xi_{2, l}, \xi_{3, l}\right)=\frac{75}{\pi} \sum_{k=1}^{N} \lambda_{k} \frac{z_{k, l}\left(x_{2, k}-\xi_{2, l}\right)\left(8 z_{k, l}^{4}-40 \rho_{k, l}^{2} z_{k, l}^{2}+15 \rho_{k, l}^{4}\right)}{\left(z_{k, l}^{2}+\rho_{k, l}^{2}\right)^{5.5}} \\
W_{y y z}\left(\xi_{l, l}, \xi_{2, l}, \xi_{3, l}\right)=\frac{75}{\pi} \sum_{k=1}^{N} \lambda_{k} \frac{z_{k, l}\left(x_{2, k}-\xi_{2, l}\right)\left(8 z_{k, l}^{4}-40 \rho_{k, l}^{2} z_{k, l}^{2}+15 \rho_{k, l}^{4}\right)}{\left(z_{k, l}^{2}+\rho_{k, l}^{2}\right)^{5.5}}
\end{gathered}
$$

Полученные выше выражения были положены в основу алгоритма и программы Gradient-F вычисления высших производных гравитационного потенциала на основе F-аппроксимации гравитационного поля.

Для оценки точности вычисления производных был использован тестовый пример (2500 точек), для которого были рассчитаны теоретические значения аномального гравитационного поля, также значения горизонтальных и вертикальных производных от 7 призматических тел. Для тестового примера была выполнена $\mathrm{F}$-аппроксимация и рассчитаны значения горизонтальных (Wxz, Wyz, Wsz) и вертикальных ( $\left.W z z, W_{z z z}\right)$, которые сравнивались с теоретическими значениями. Результаты оценки точности вычисления производных приведены в таблице 1. По результатам расчетов были построены карты производных, а также карты разностных полей между теоретическими и полученными по результатам F-аппроксимации значениями производных.

Расчеты горизонтальных и вертикальных производных по результатам $\mathrm{F}$-аппроксимации были также выполнены для модельных примеров №1, №2 и №3. Результаты оценки точности приведены в таблицах 2-4.

На рис. 1-24 приведены карты теоретических и расчетных на основе F-аппроксимации значений горизонтальных и вертикальных производных, а также карты разностных полей для производных $W s z, W z z$ и $W z z z$ для модельных примеров № 1, № 2 и № 3 .

На рис. 25-26 представлены карты $W z z$ и $W z z z$ для Сереговской и Сибирской площадей. Из анализа карт разностных полей видно, что максимальные отклонения отмечаются на периферии. Точность вычисления высших производных без учета около $5 \%$ периферийных точек значительно выше для всех точек (табл. 1-4).

Таблича 1.

\begin{tabular}{|c|c|c|c|c|}
\hline \multirow{2}{*}{ Элемент поля } & \multicolumn{4}{|c|}{$\gamma=\frac{\left\|W_{u c m}-W_{\text {вьи }}\right\|_{E}}{\left\|W_{\text {ист }}\right\|_{E}}$} \\
\cline { 2 - 5 } & \multicolumn{3}{|c|}{$\mathrm{C}^{2}=10^{-5}$} & \multicolumn{2}{c|}{$\mathrm{C}^{2}=10^{-3}$} \\
\cline { 2 - 5 } & $\mathrm{K}_{\text {конт }}=2500$ & $\mathrm{~K}_{\text {конт }}=1805$ & $\mathrm{~K}_{\text {конт }}=2500$ & $\mathrm{~K}_{\text {конт }}=1805$ \\
\hline$W z$ & 0.00214 & 0.00126 & 0.01504 & 0.01211 \\
\hline$W x z$ & 0.05918 & 0.00794 & 0.12531 & 0.04282 \\
\hline$W y z$ & 0.05056 & 0.00567 & 0.11437 & 0.02739 \\
\hline$W s z$ & 0.05164 & 0.00527 & 0.11271 & 0.02526 \\
\hline$W z z$ & 0.19379 & 0.05757 & 0.22543 & 0.06614 \\
\hline$W z z z$ & 0.32302 & 0.03943 & 0.43675 & 0.11079 \\
\hline
\end{tabular}


Таблиияа 2.

\begin{tabular}{|c|c|c|c|c|c|c|c|c|c|c|c|c|c|}
\hline \multirow{2}{*}{$\begin{array}{c}\text { Элемент } \\
\text { поля }\end{array}$} & \multicolumn{4}{|c|}{$\mathrm{C}^{2}=10^{-5}$} & \multicolumn{4}{c|}{$\mathrm{C}^{2}=10^{-4}$} & \multicolumn{5}{c|}{$\mathrm{C}^{2}=10^{-3}$} \\
\cline { 2 - 14 } & $\mathrm{K}_{\text {конт }}=6000$ & \multicolumn{2}{|c|}{$\mathrm{K}_{\text {конт }}=4914$} & \multicolumn{2}{|c|}{$\mathrm{K}_{\text {конт }}=6000$} & \multicolumn{2}{|c|}{$\mathrm{K}_{\text {конт }}=4914$} & \multicolumn{2}{|c|}{$\mathrm{K}_{\text {конт }}=6000$} & \multicolumn{2}{|c|}{$\mathrm{K}_{\text {конт }}=4914$} \\
\cline { 2 - 14 } & $\gamma$ & $\sigma$ & $\gamma$ & $\sigma$ & $\gamma$ & $\sigma$ & $\gamma$ & $\sigma$ & $\gamma$ & $\sigma$ & $\gamma$ & $\sigma$ \\
\hline$W z$ (mgal) & 0.002 & 0.01 & 0.001 & 0.01 & 0.006 & 0.04 & 0.002 & 0.02 & 0.016 & 0.11 & 0.009 & 0.08 \\
\hline$W x z(E)$ & 0.289 & 0.94 & 0.010 & 0.09 & 0.433 & 1.41 & 0.018 & 0.17 & 0.627 & 2.04 & 0.044 & 0.42 \\
\hline$W y z(E)$ & 0.175 & 0.47 & 0.007 & 0.06 & 0.290 & 0.77 & 0.014 & 0.14 & 0.464 & 1.23 & 0.033 & 0.31 \\
\hline$W s z(E)$ & 0.245 & 1.03 & 0.010 & 0.10 & 0.369 & 1.55 & 0.019 & 0.18 & 0.544 & 2.29 & 0.044 & 0.42 \\
\hline$W z z(E)$ & 1.00 & 4.50 & 0.145 & 1.38 & 1.088 & 4.89 & 0.152 & 1.45 & 1.172 & 5.27 & 0.159 & 1.52 \\
\hline$W z z z \times 10^{-15}$ & 2.48 & 23.6 & 0.203 & 1.9 & 2.957 & 28.2 & 0.327 & 3.1 & 3.403 & 32.5 & 0.599 & 5.7 \\
\hline
\end{tabular}

Таблицุа 3.

\begin{tabular}{|c|c|c|c|c|c|c|c|c|c|c|c|c|c|}
\hline \multirow{2}{*}{$\begin{array}{c}\text { Элемент } \\
\text { поля }\end{array}$} & \multicolumn{4}{|c|}{$\mathrm{C}^{2}=10^{-5}$} & \multicolumn{4}{c|}{$\mathrm{C}^{2}=10^{-4}$} & \multicolumn{5}{c|}{$\mathrm{C}^{2}=10^{-3}$} \\
\cline { 2 - 14 } & $\mathrm{K}_{\text {конт }}=6000$ & $\mathrm{~K}_{\text {конт }}=4914$ & \multicolumn{2}{|c|}{$\mathrm{K}_{\text {конт }}=6000$} & \multicolumn{2}{|c|}{$\mathrm{K}_{\text {конт }}=4914$} & \multicolumn{2}{|c|}{$\mathrm{K}_{\text {конт }}=6000$} & \multicolumn{2}{|c|}{$\mathrm{K}_{\text {конт }}=4914$} \\
\cline { 2 - 15 } & $\gamma$ & $\sigma$ & $\gamma$ & $\sigma$ & $\gamma$ & $\sigma$ & $\gamma$ & $\sigma$ & $\gamma$ & $\sigma$ & $\gamma$ & $\sigma$ \\
\hline$W z$ (mgal) & 0.002 & 0.01 & 0.001 & 0.01 & 0.005 & 0.02 & 0.001 & 0.02 & 0.015 & 0.06 & 0.004 & 0.05 \\
\hline$W x z(E)$ & 0.129 & 0.45 & 0.013 & 0.05 & 0.189 & 0.66 & 0.008 & 0.11 & 0.256 & 0.90 & 0.017 & 0.22 \\
\hline$W y z(E)$ & 0.064 & 0.21 & 0.010 & 0.03 & 0.102 & 0.33 & 0.006 & 0.07 & 0.155 & 0.50 & 0.013 & 0.17 \\
\hline$W s z(E)$ & 0.100 & 0.48 & 0.009 & 0.04 & 0.148 & 0.71 & 0.008 & 0.11 & 0.204 & 0.97 & 0.016 & 0.21 \\
\hline$W z z(E)$ & 0.370 & 1.81 & 0.108 & 0.53 & 0.400 & 1.96 & 0.043 & 0.56 & 0.424 & 2.08 & 0.047 & 0.61 \\
\hline$W z z z \times 10^{-15}$ & 0.783 & 10.2 & 0.076 & 0.99 & 0.915 & 11.9 & 0.127 & 1.65 & 0.915 & 13.2 & 0.237 & 3.08 \\
\hline
\end{tabular}

Таблицุа 4.

\begin{tabular}{|c|c|c|c|c|c|c|c|c|c|c|c|c|c|}
\hline \multirow{2}{*}{$\begin{array}{c}\text { Элемент } \\
\text { поля }\end{array}$} & \multicolumn{4}{|c|}{$\mathrm{C}^{2}=10^{-5}$} & \multicolumn{4}{c|}{$\mathrm{C}^{2}=10^{-4}$} & \multicolumn{5}{c|}{$\mathrm{C}^{2}=10^{-3}$} \\
\cline { 2 - 13 } & $\mathrm{K}_{\text {конт }}=6000$ & \multicolumn{2}{|c|}{$\mathrm{K}_{\text {конт }}=4991$} & \multicolumn{2}{|c|}{$\mathrm{K}_{\text {конт }}=6000$} & \multicolumn{2}{|c|}{$\mathrm{K}_{\text {конт }}=4991$} & \multicolumn{2}{|c|}{$\mathrm{K}_{\text {конт }}=6000$} & \multicolumn{2}{|c|}{$\mathrm{K}_{\text {конт }}=4991$} \\
\cline { 2 - 14 } & $\gamma$ & $\sigma$ & $\gamma$ & $\sigma$ & $\gamma$ & $\sigma$ & $\gamma$ & $\sigma$ & $\gamma$ & $\sigma$ & $\gamma$ & $\sigma$ \\
\hline$W z$ (mgal) & 0.002 & 0.01 & 0.000 & 0.01 & 0.005 & 0.03 & 0.001 & 0.02 & 0.014 & 0.08 & 0.002 & 0.05 \\
\hline$W x z(E)$ & 0.112 & 0.60 & 0.006 & 0.14 & 0.228 & 1.21 & 0.013 & 0.34 & 0.400 & 2.12 & 0.025 & 0.64 \\
\hline$W y z(E)$ & 0.083 & 0.34 & 0.005 & 0.12 & 0.178 & 0.73 & 0.010 & 0.25 & 0.332 & 1.36 & 0.021 & 0.52 \\
\hline$W s z(E)$ & 0.099 & 0.66 & 0.006 & 0.15 & 0.201 & 1.35 & 0.014 & 0.35 & 0.355 & 2.38 & 0.026 & 0.64 \\
\hline$W z z(E)$ & 0.498 & 3.61 & 0.056 & 1.42 & 0.586 & 4.25 & 0.060 & 1.51 & 0.680 & 4.93 & 0.065 & 1.63 \\
\hline$W z z z x 10^{-15}$ & 1.069 & 26.9 & 0.189 & 4.8 & 1.566 & 39.4 & 0.347 & 8.7 & 2.006 & 50.5 & 0.633 & 15.9 \\
\hline
\end{tabular}




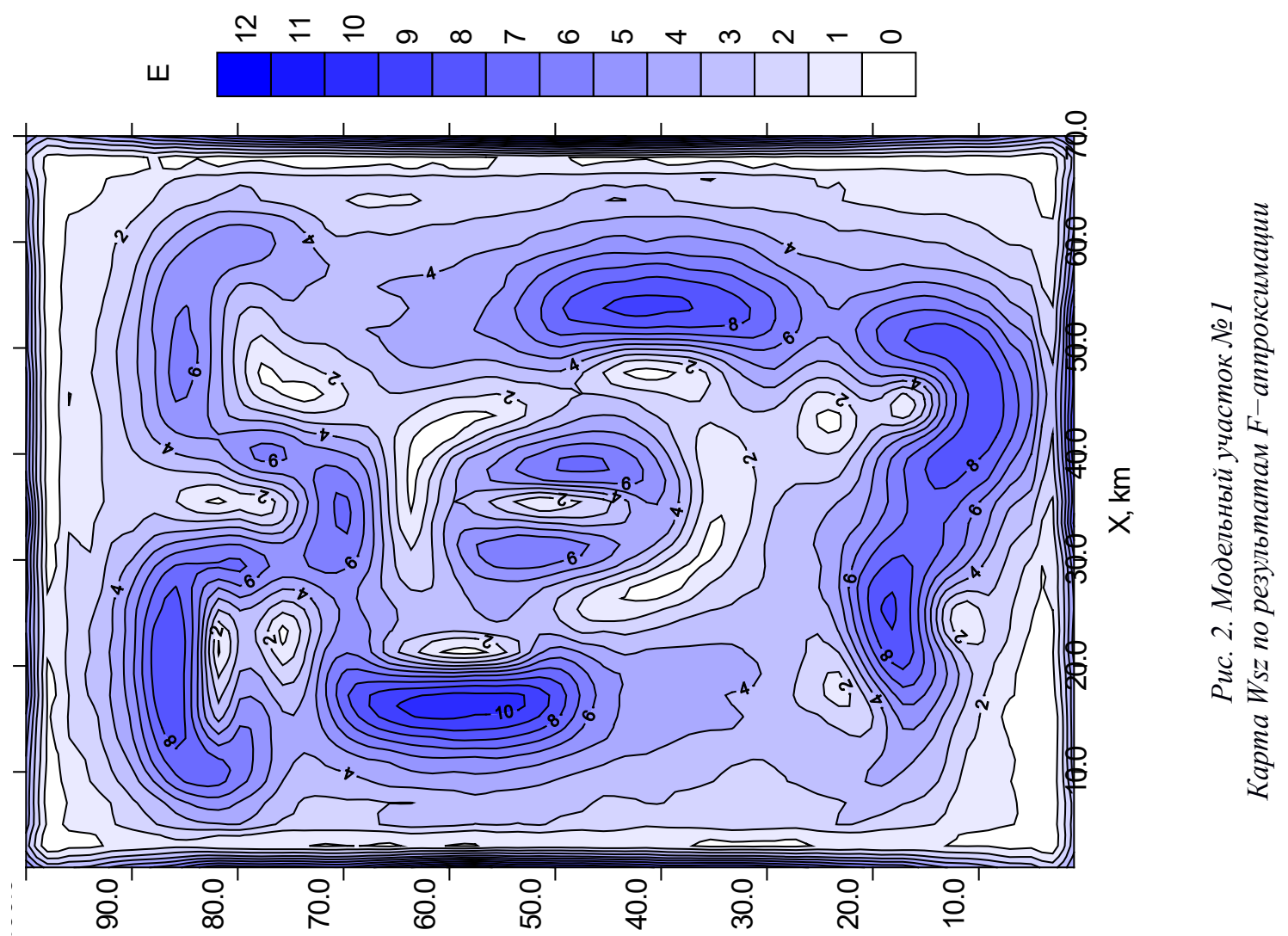

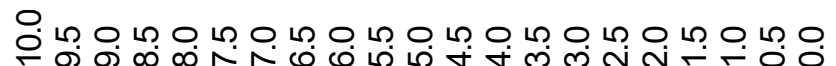

ш

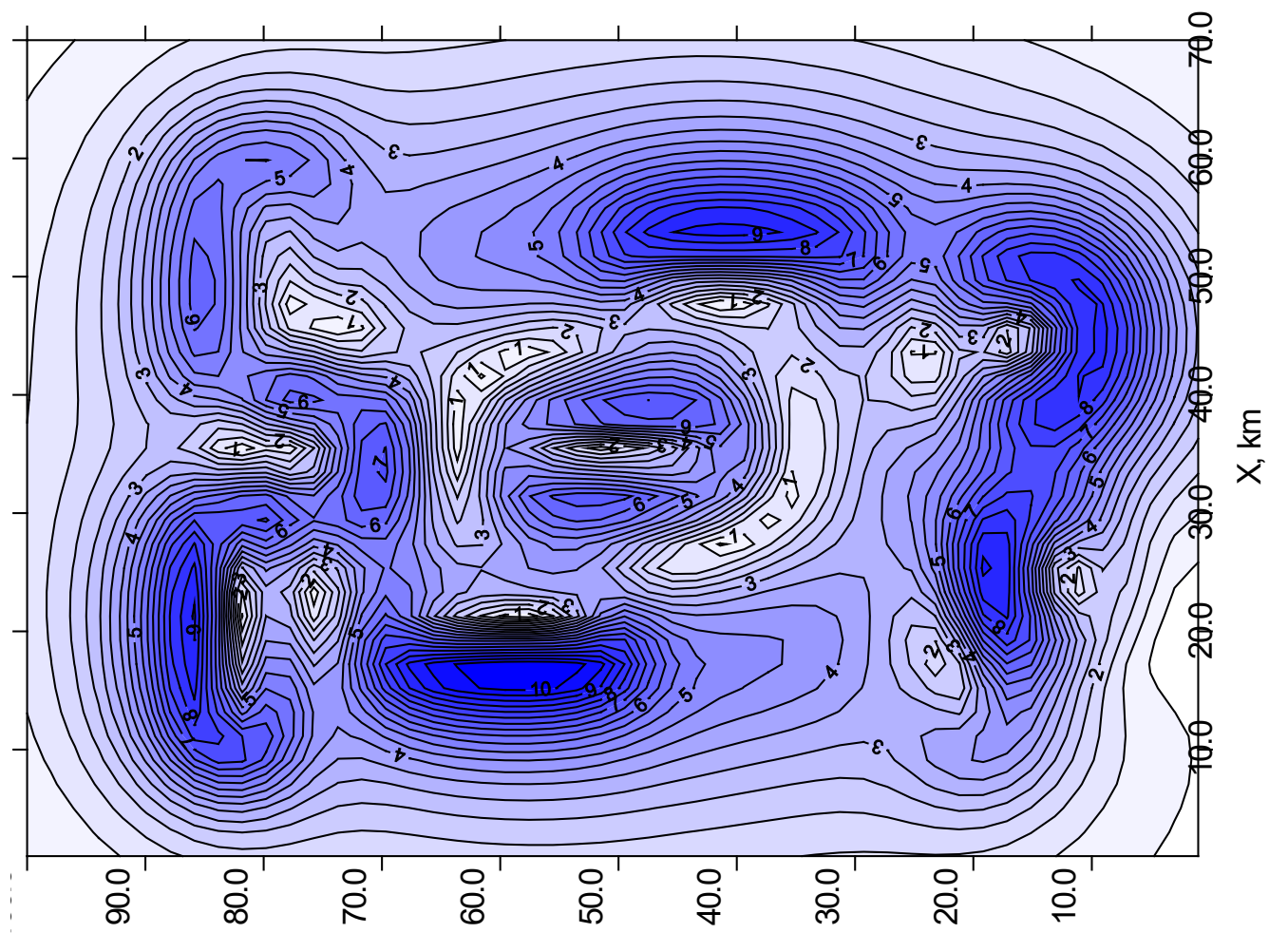

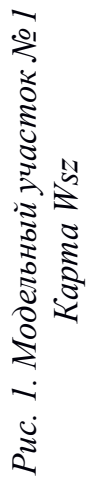




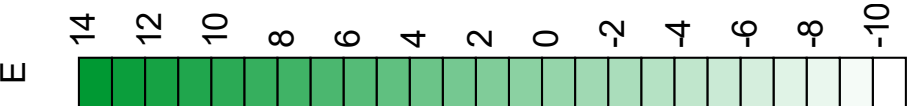

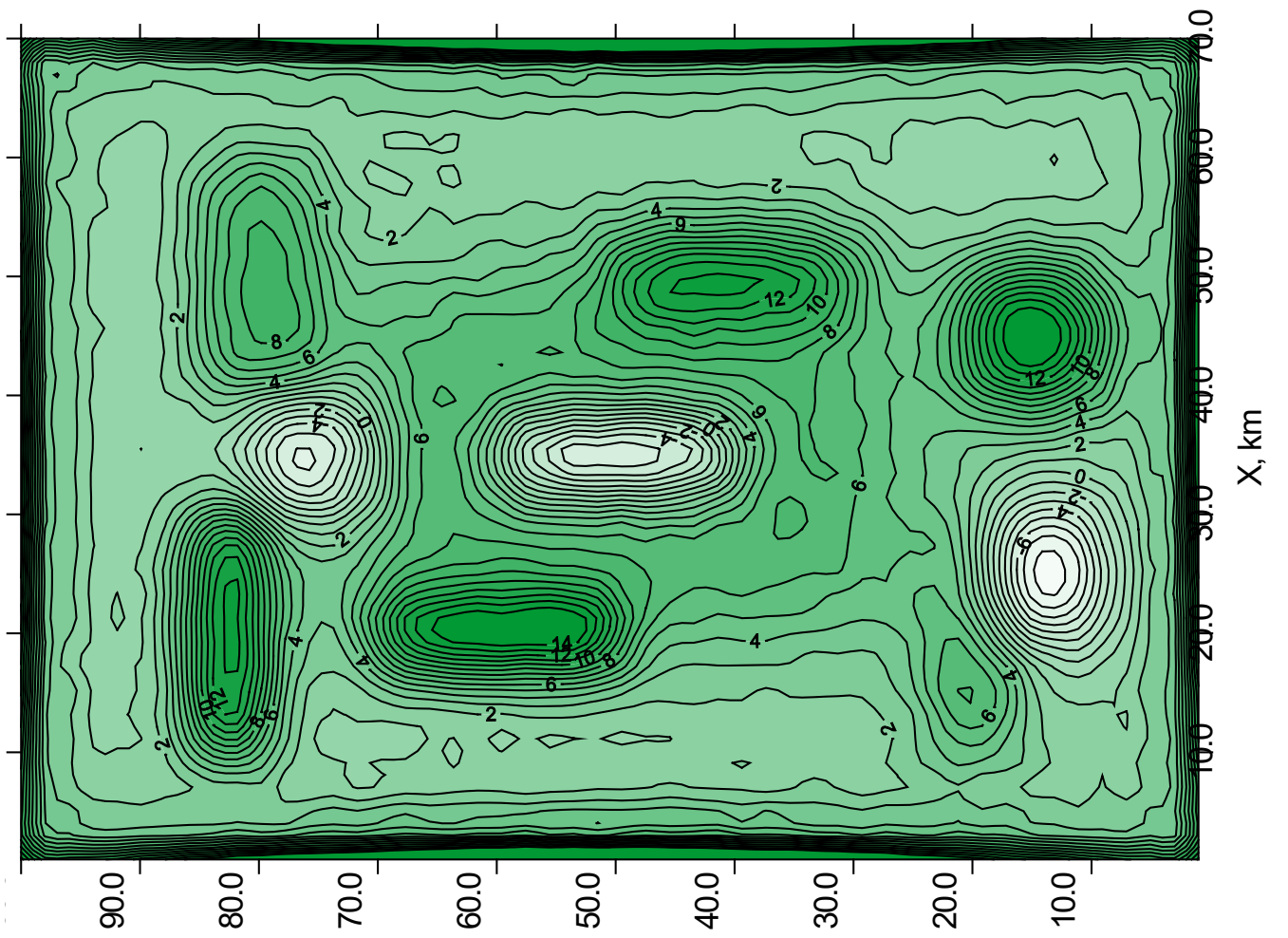

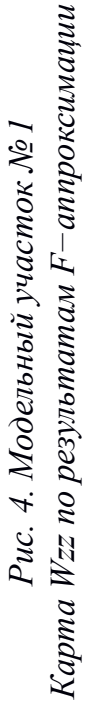

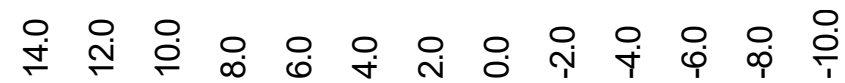

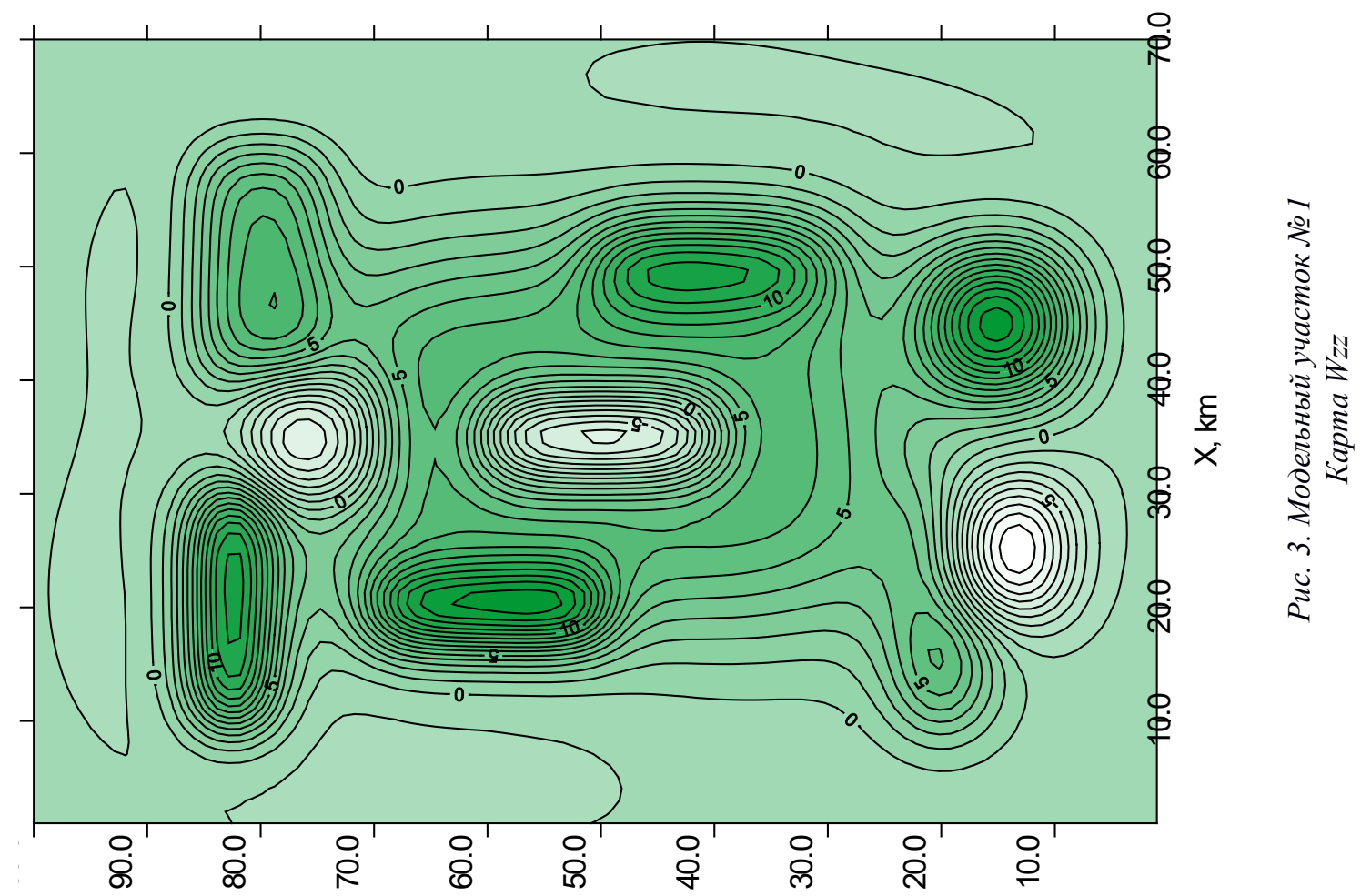




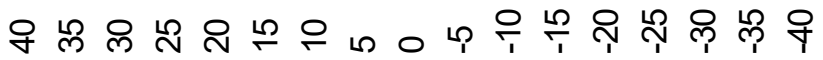

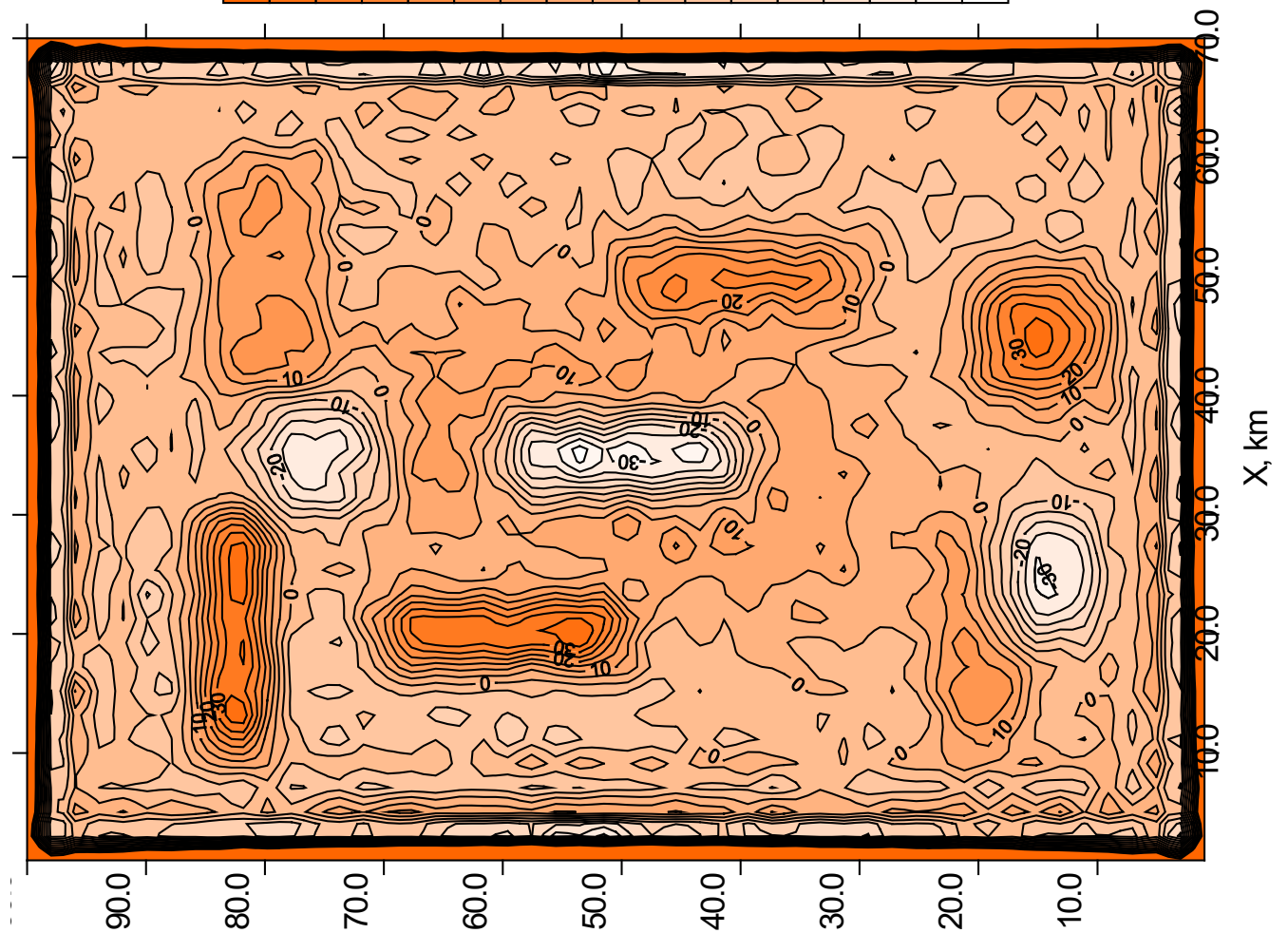

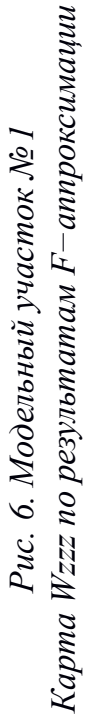

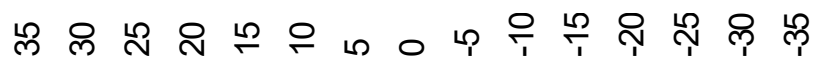

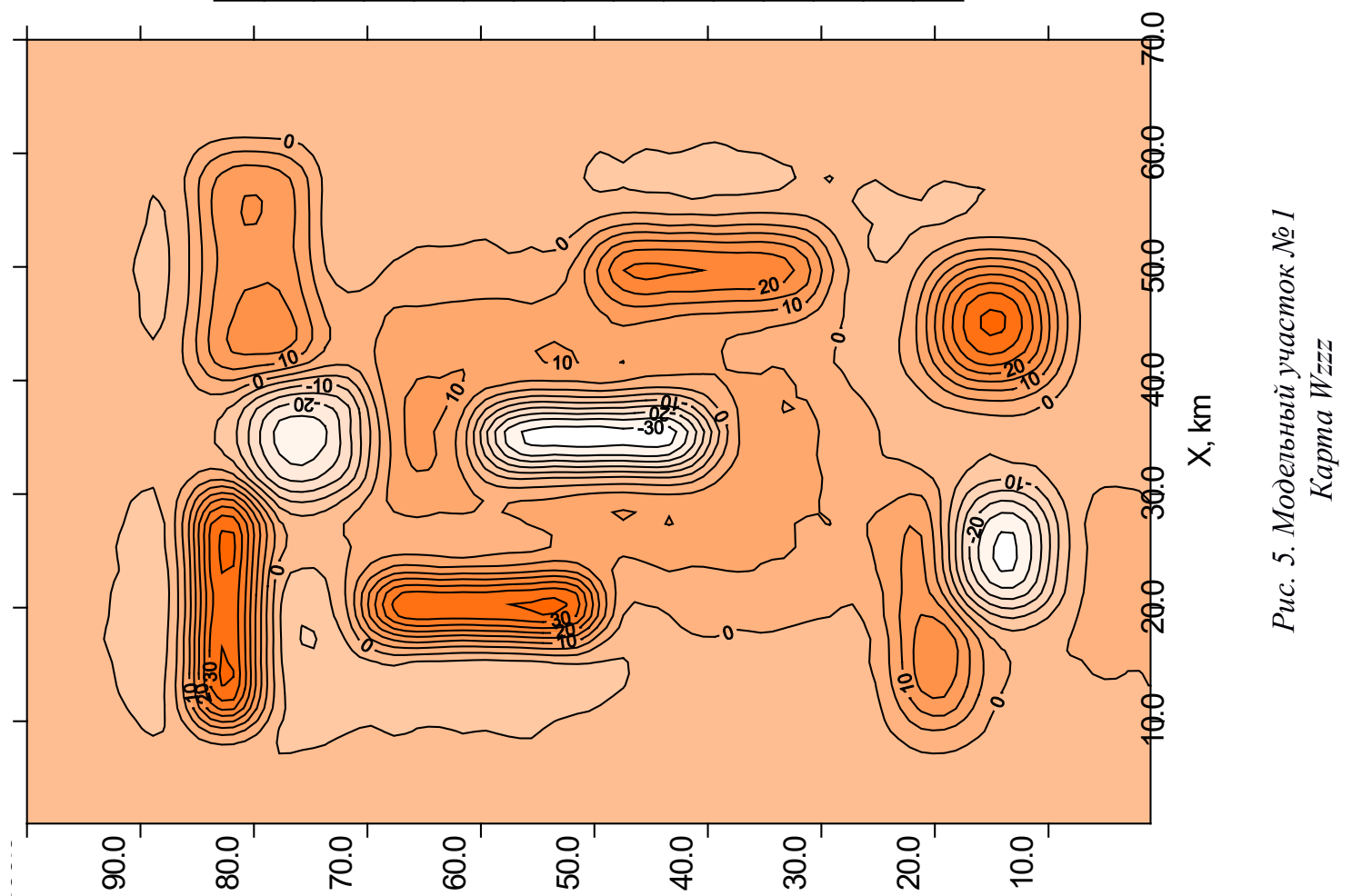




노 는
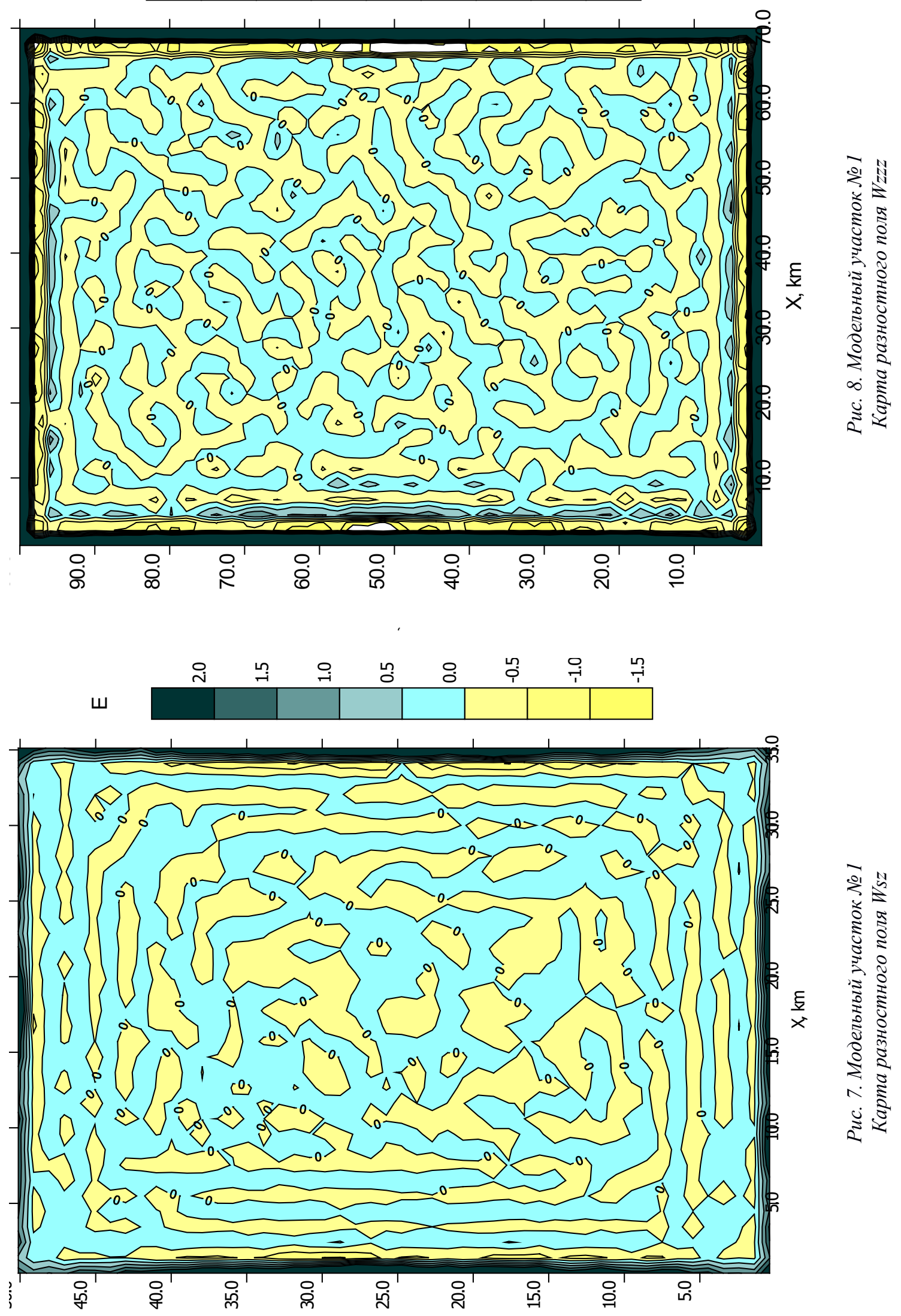
婇

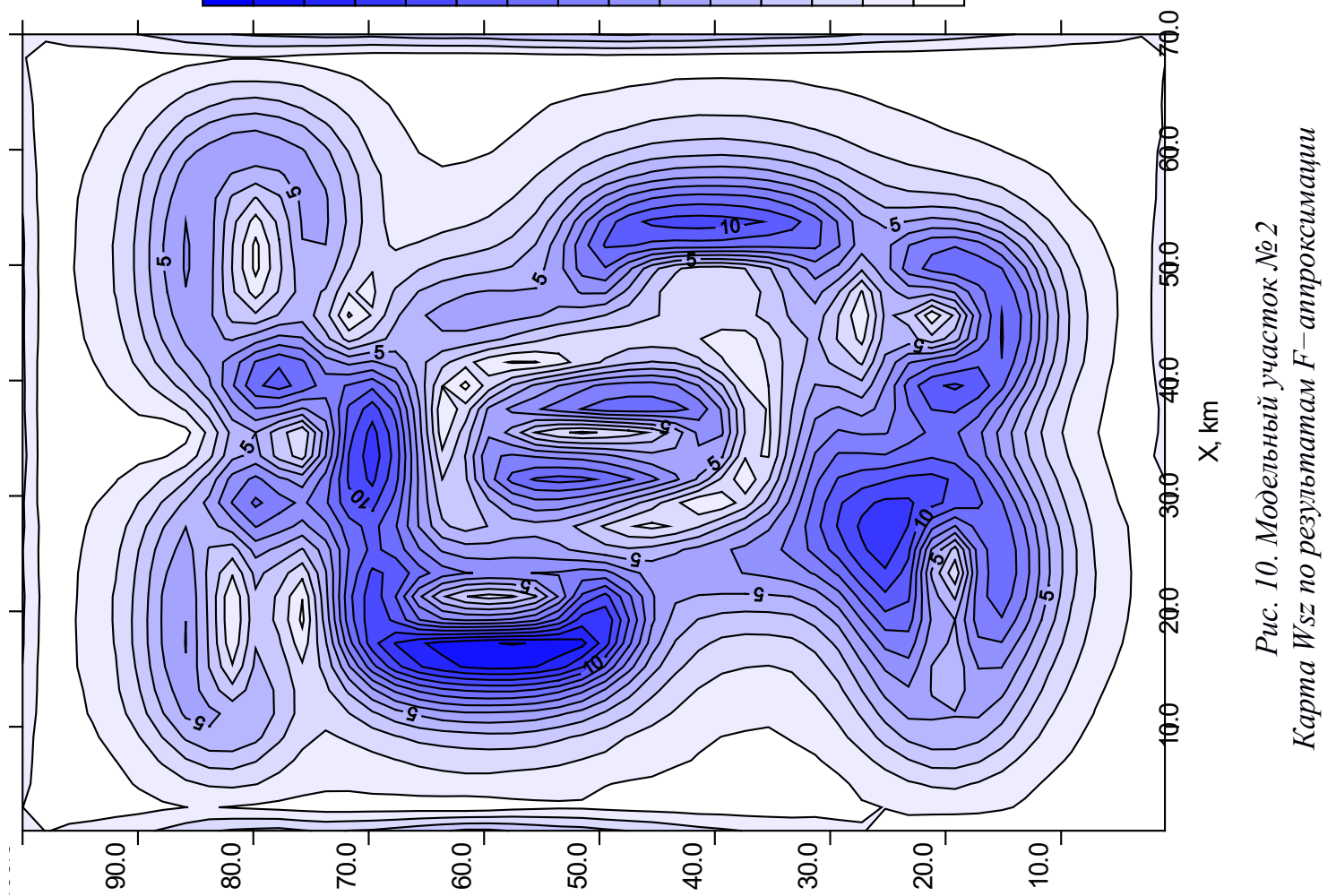

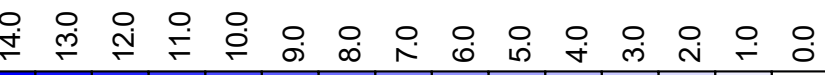

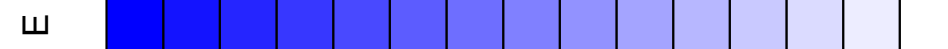

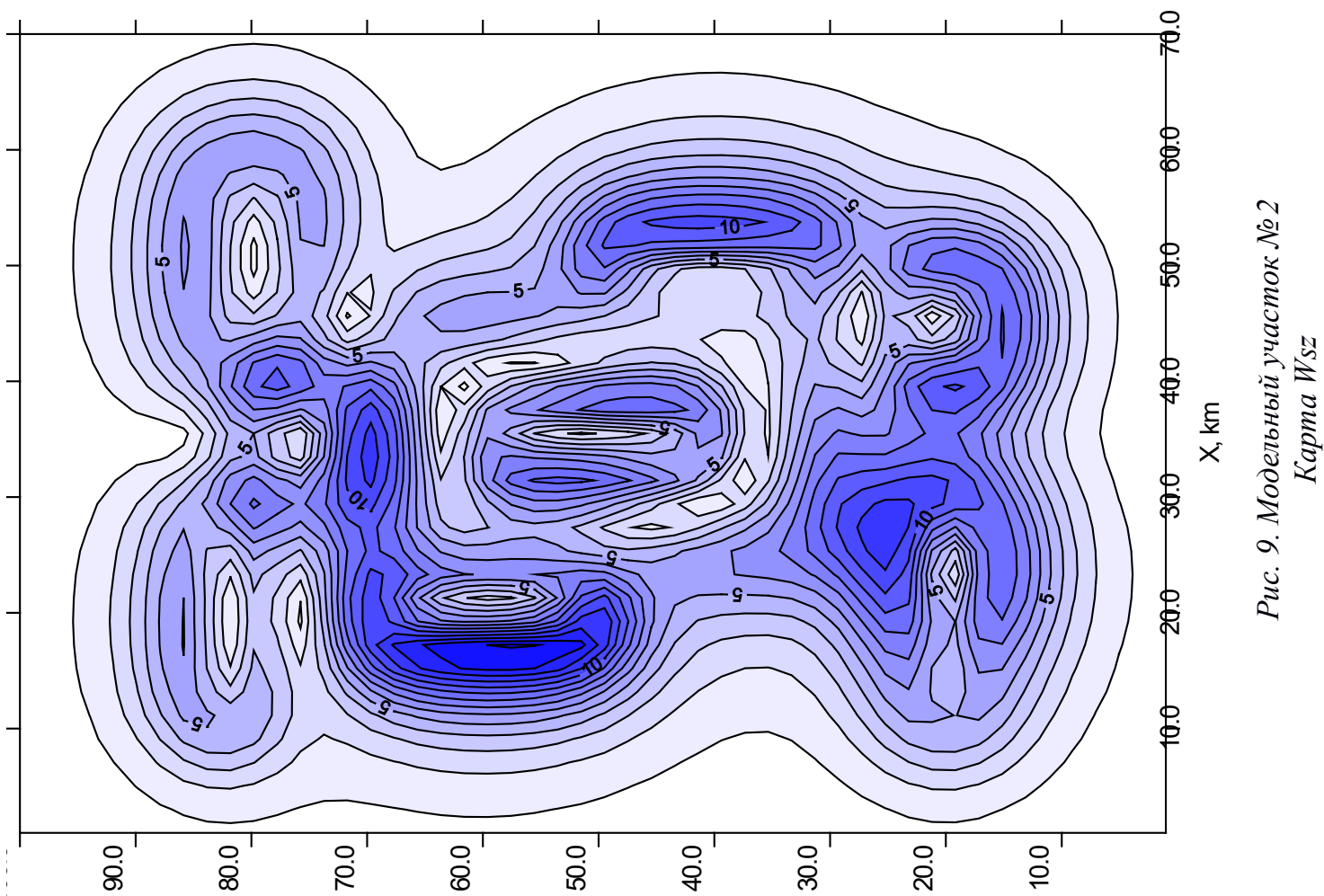




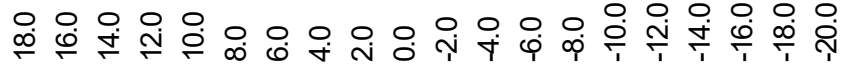

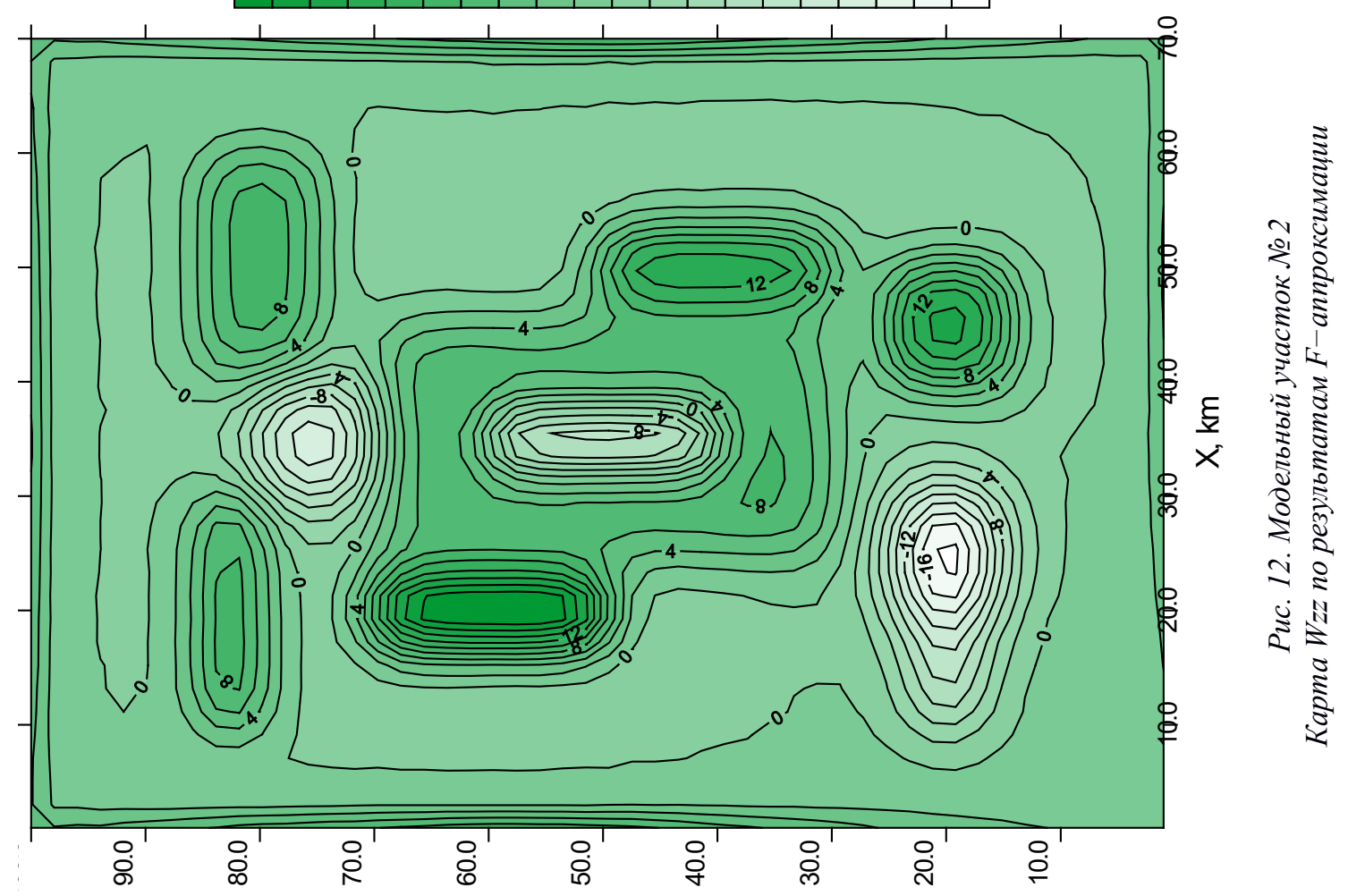

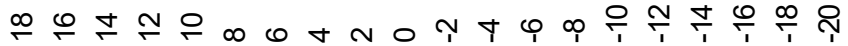

ш

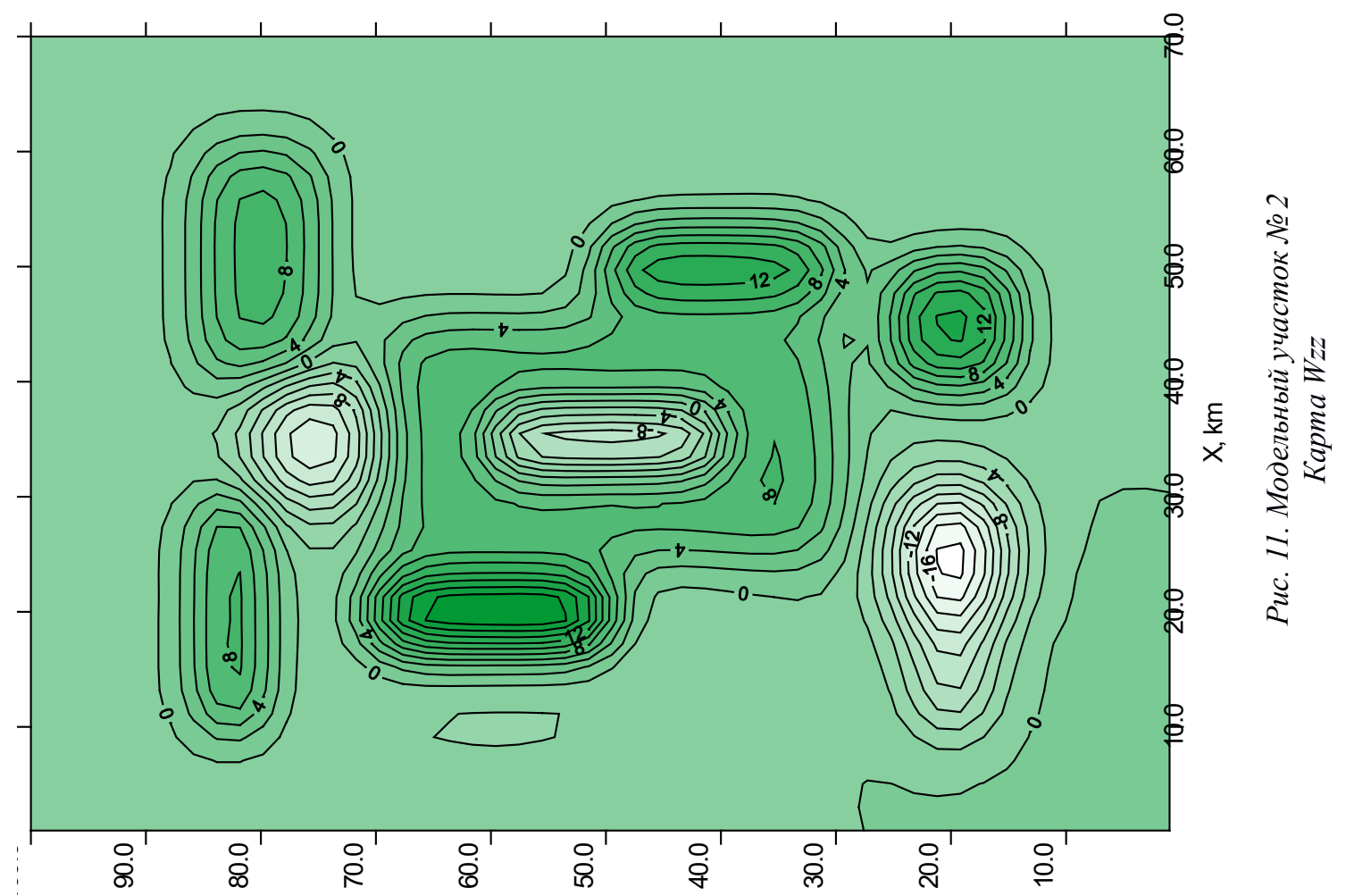




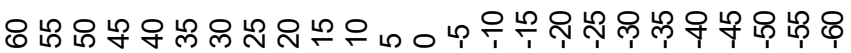

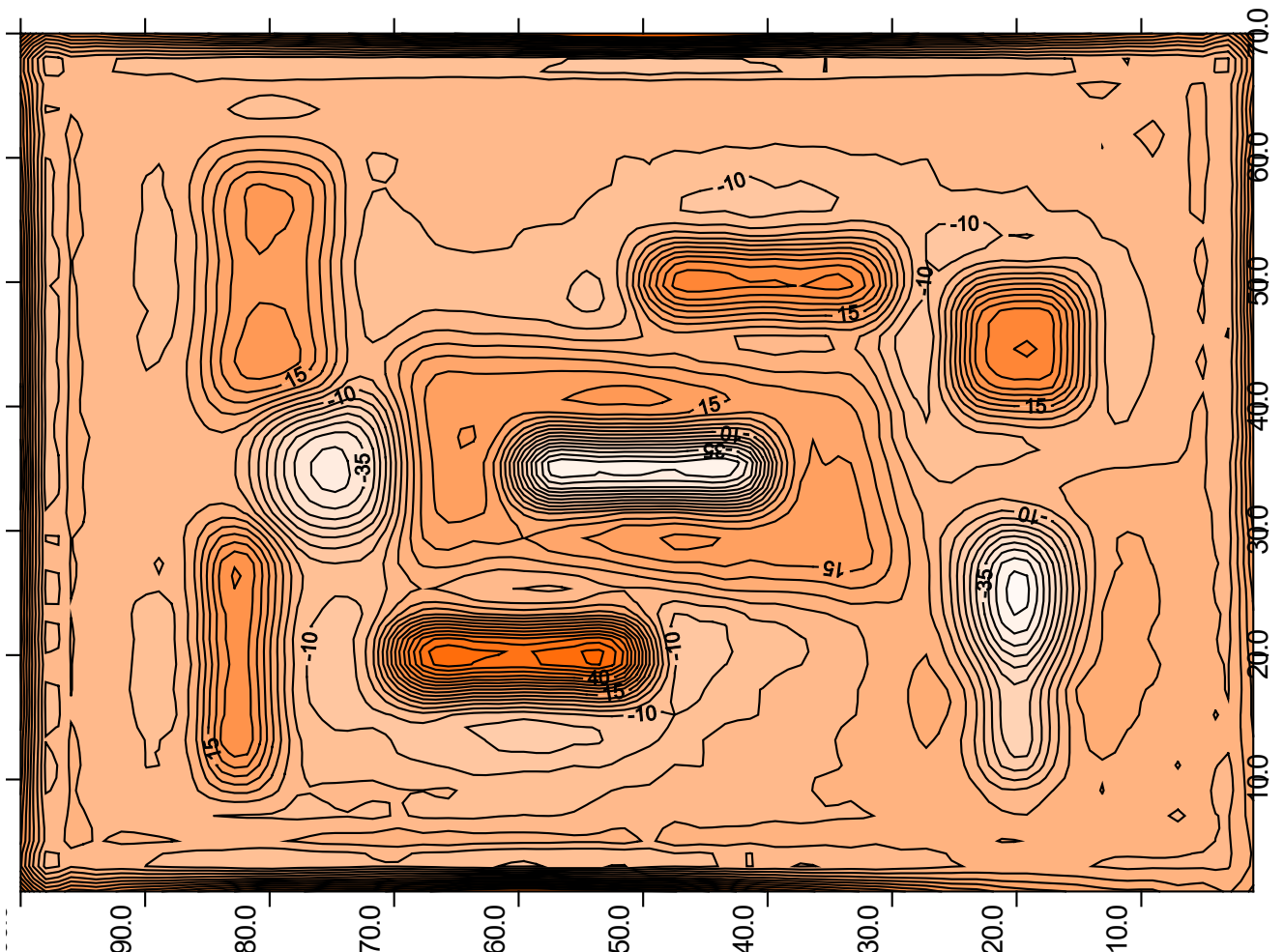

$\sim$

急

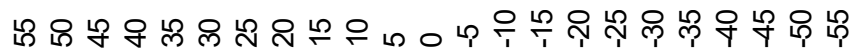

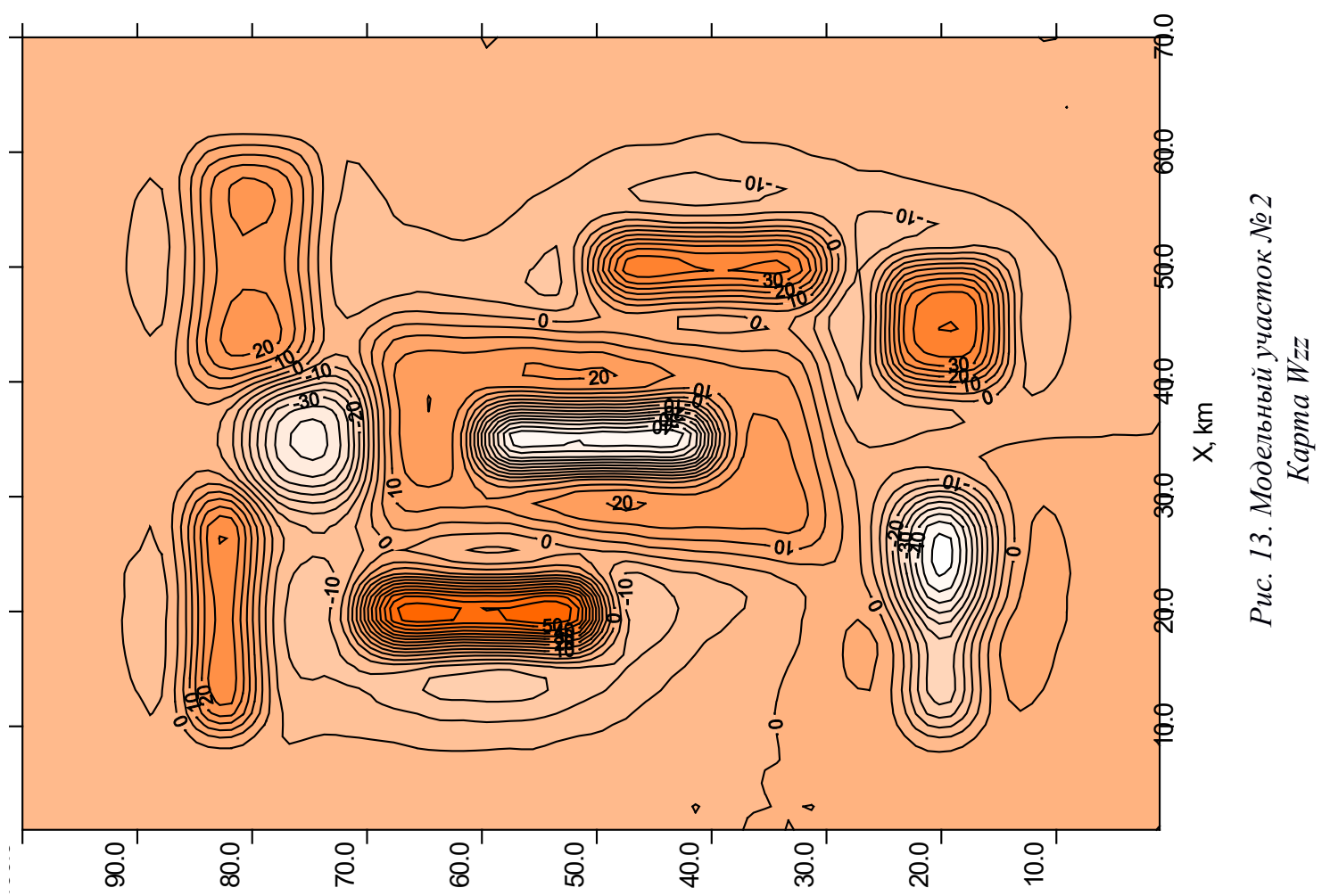




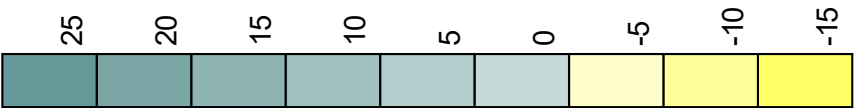

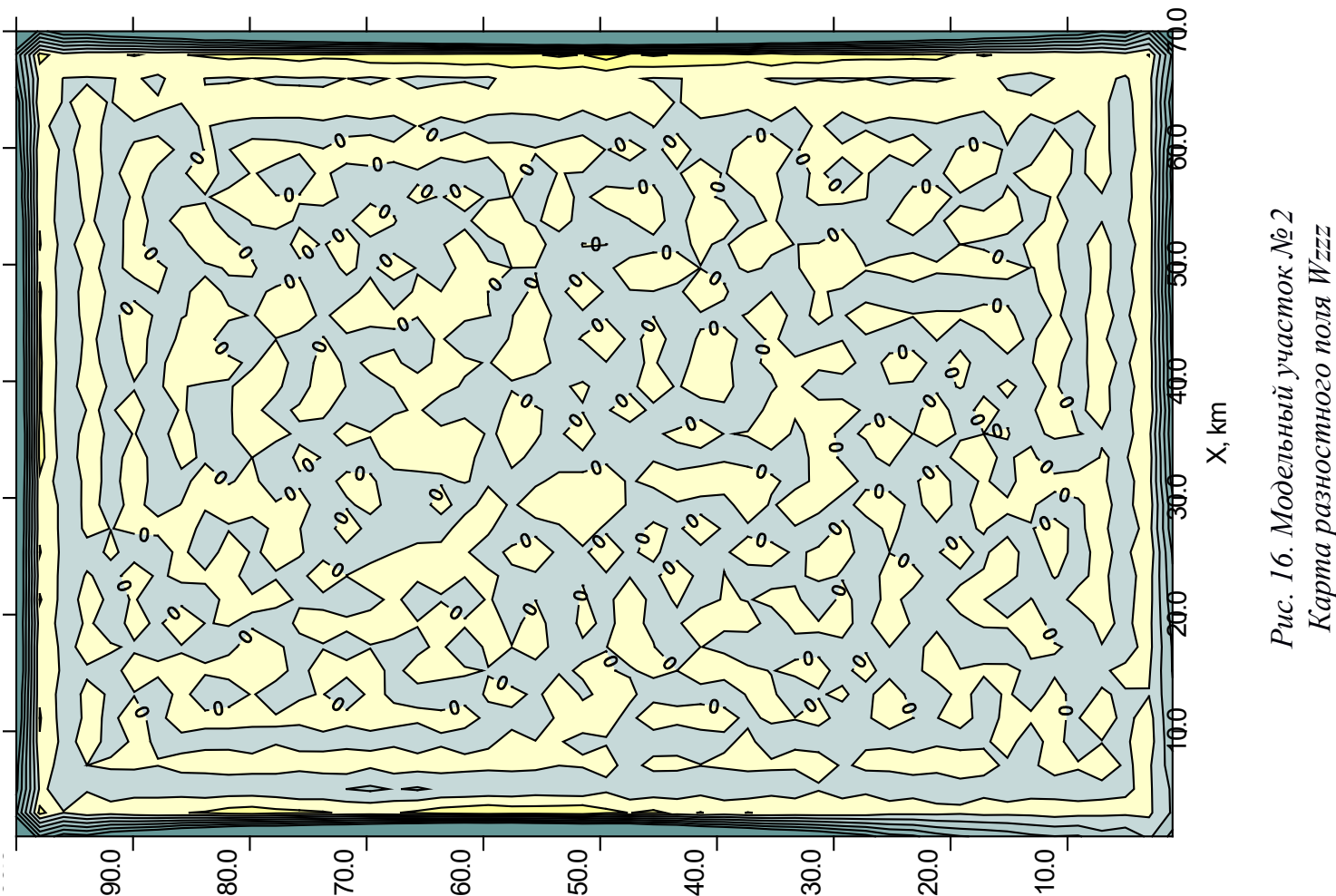

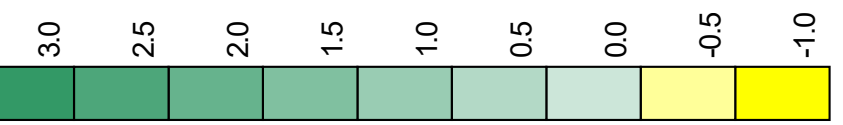

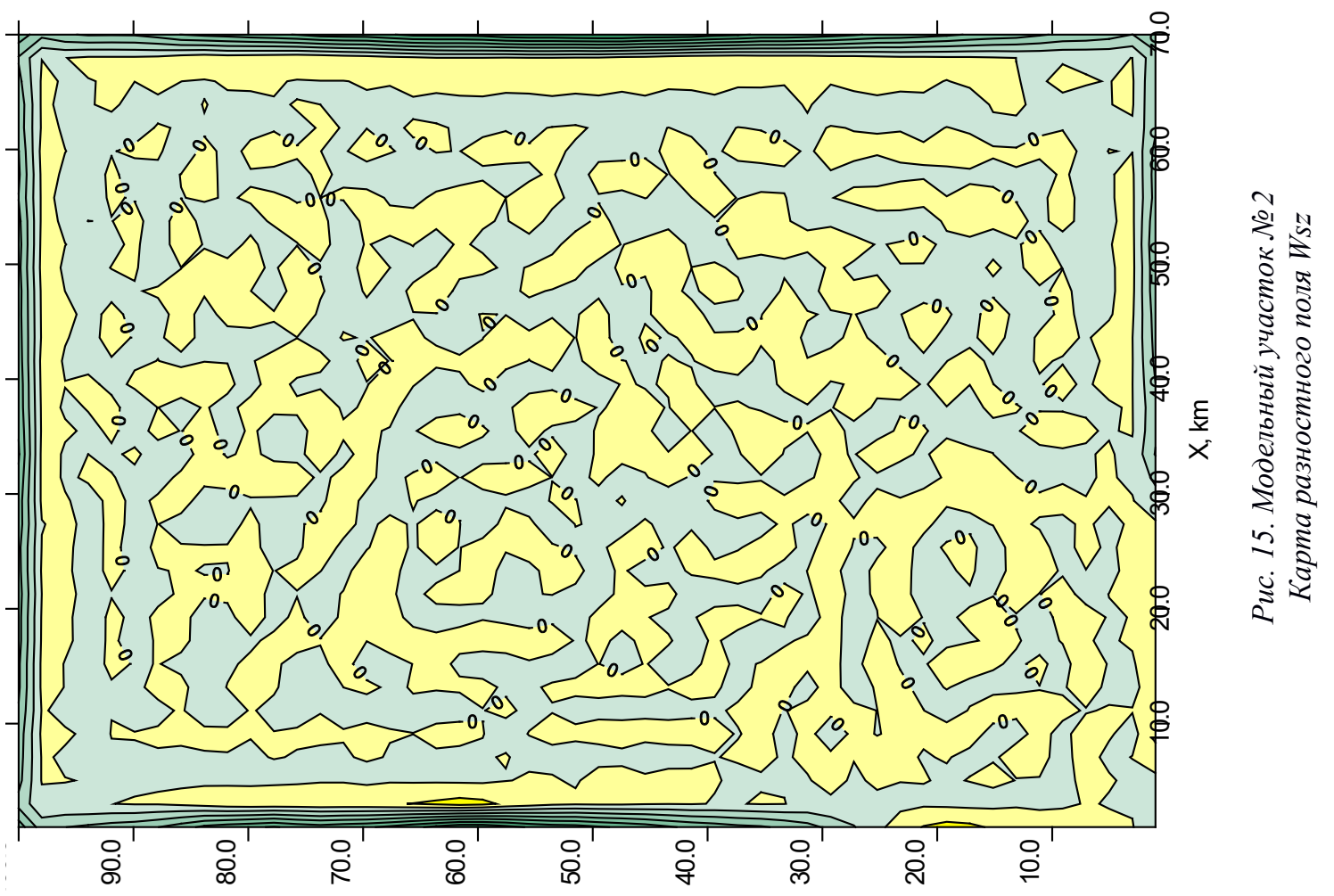


떠 믹

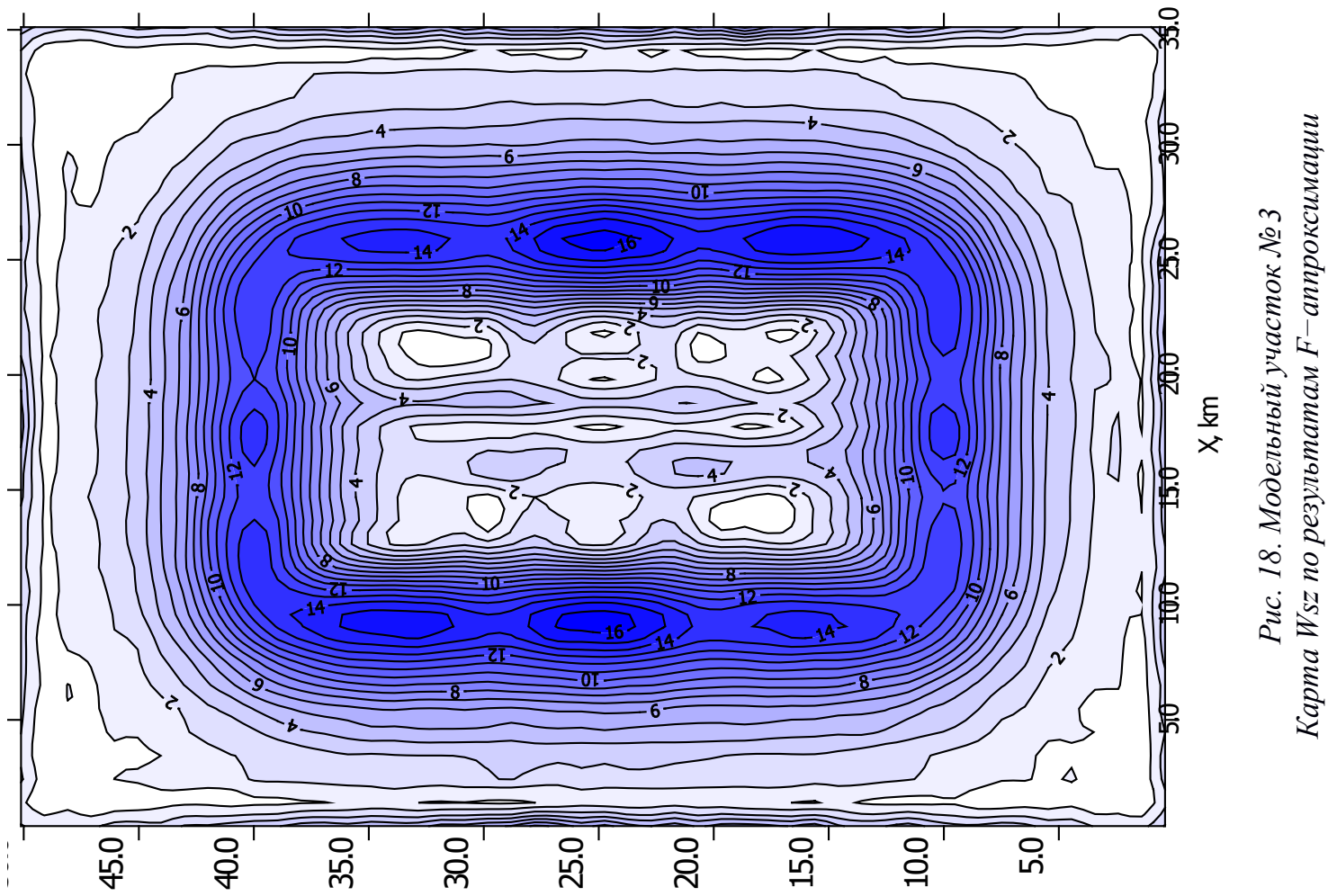

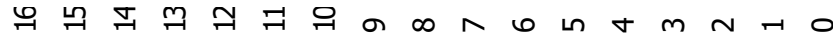

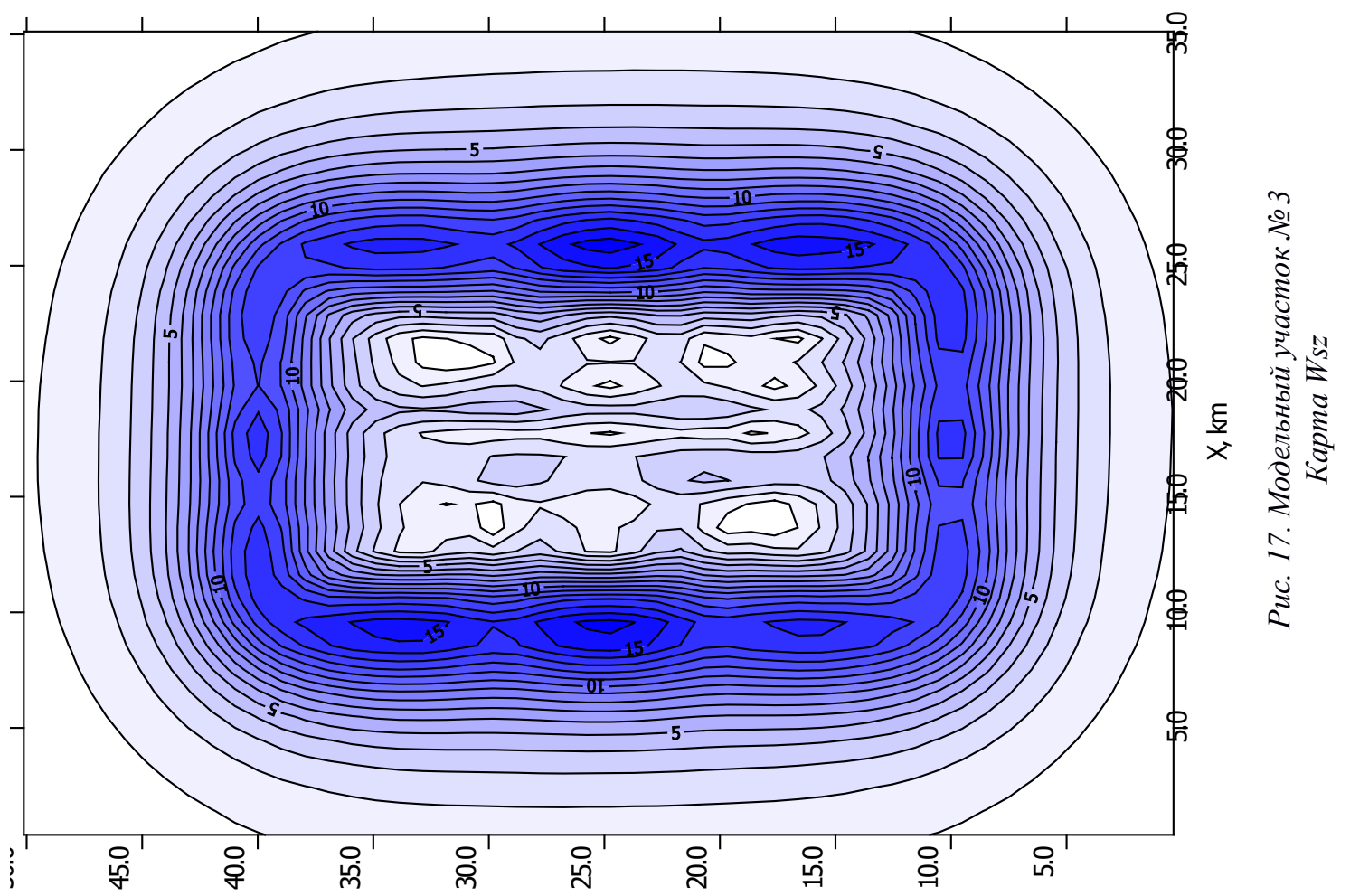




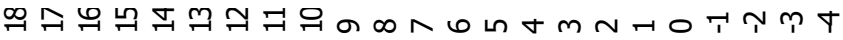

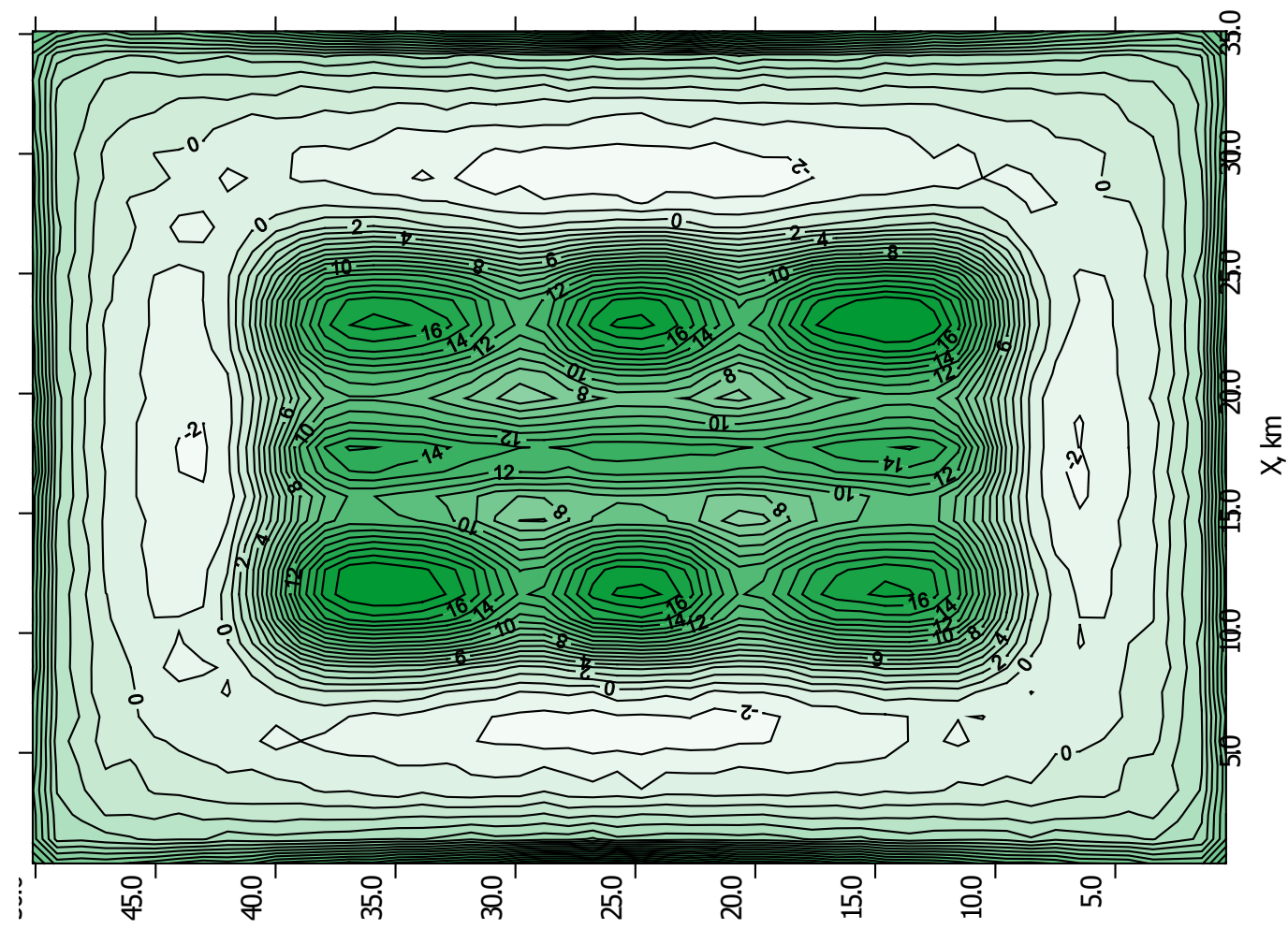

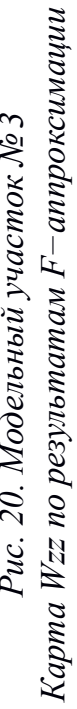

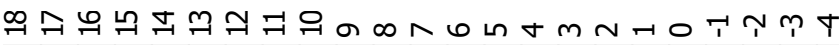

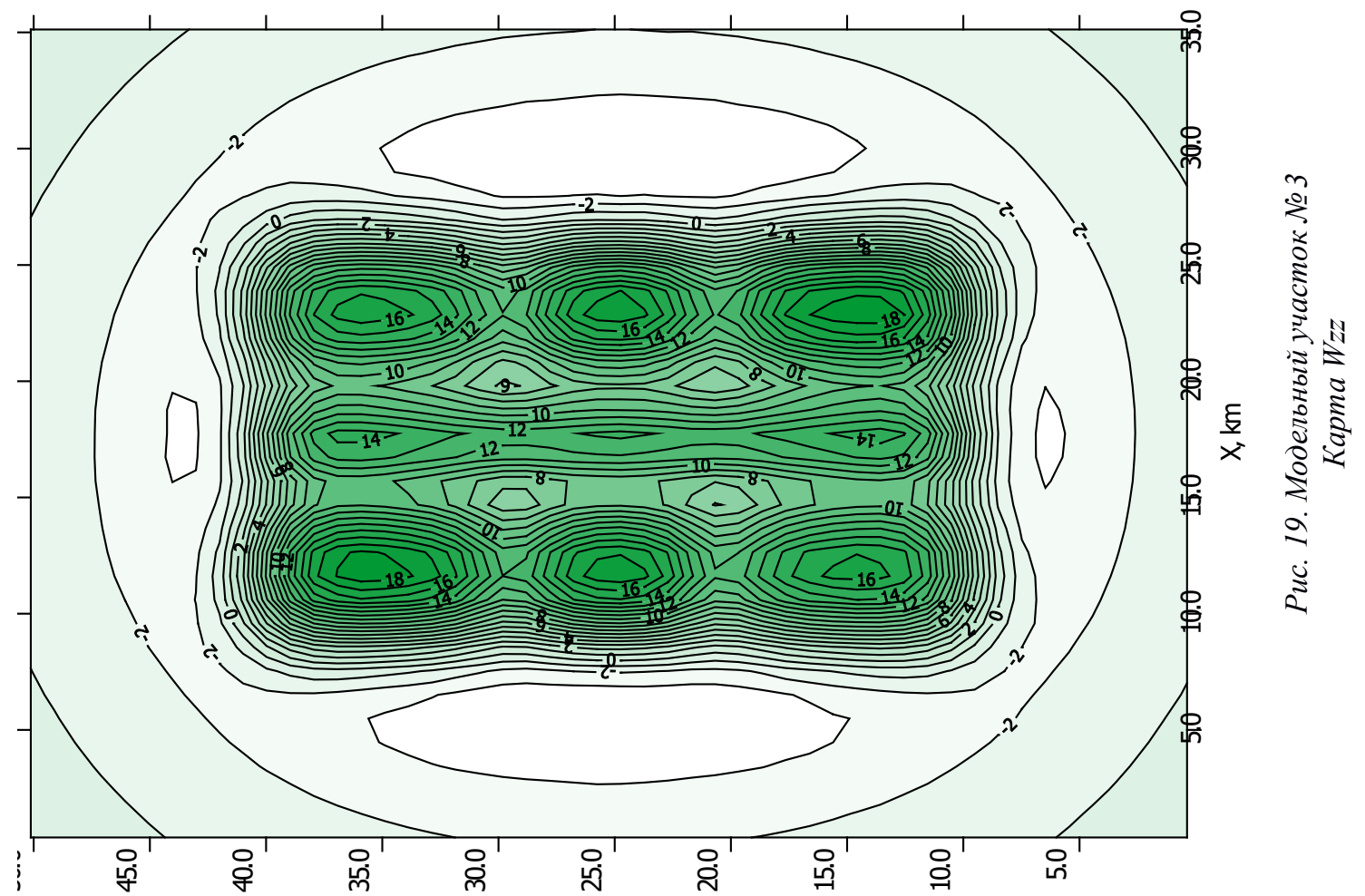




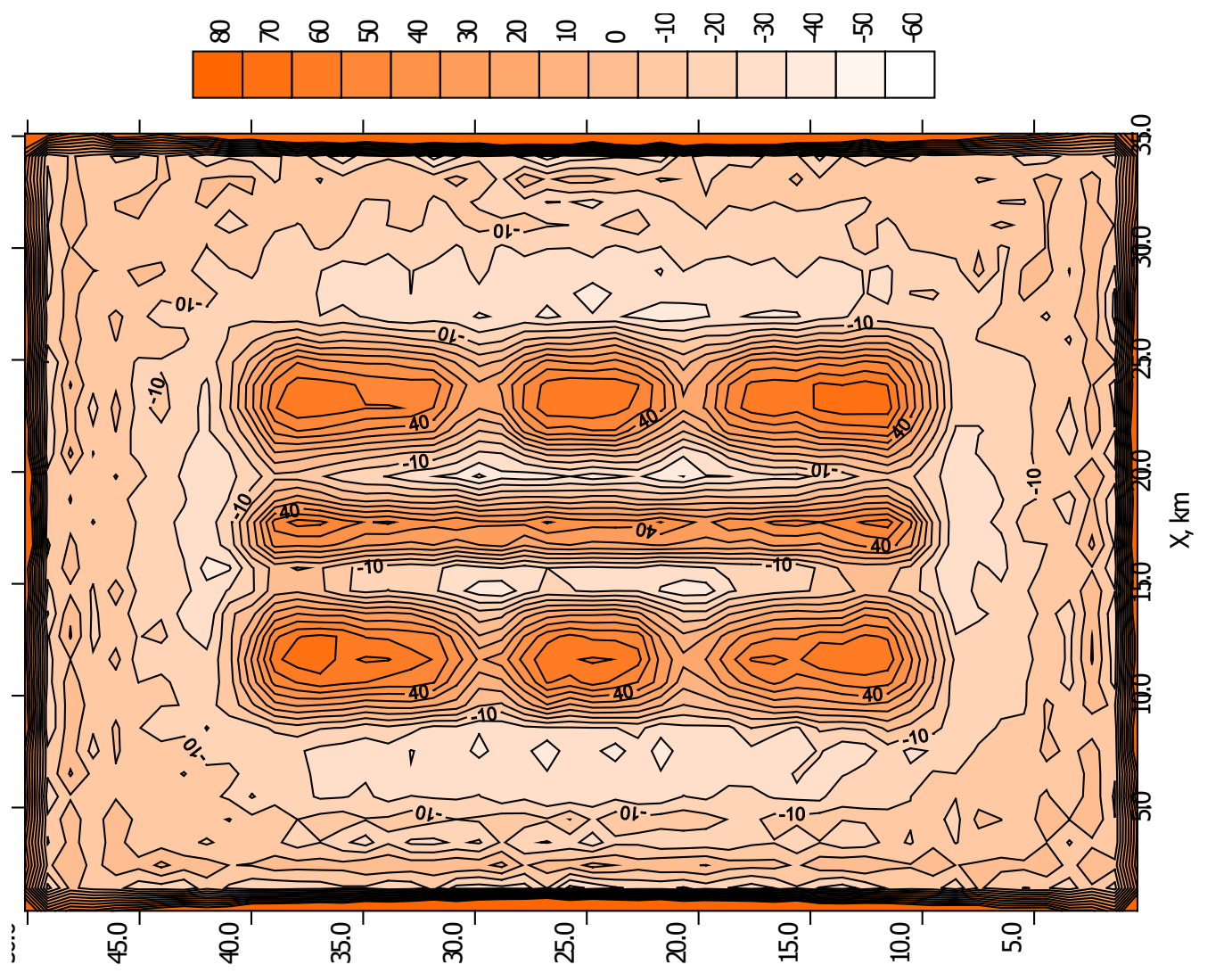

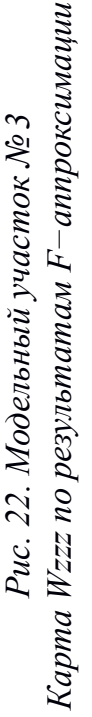

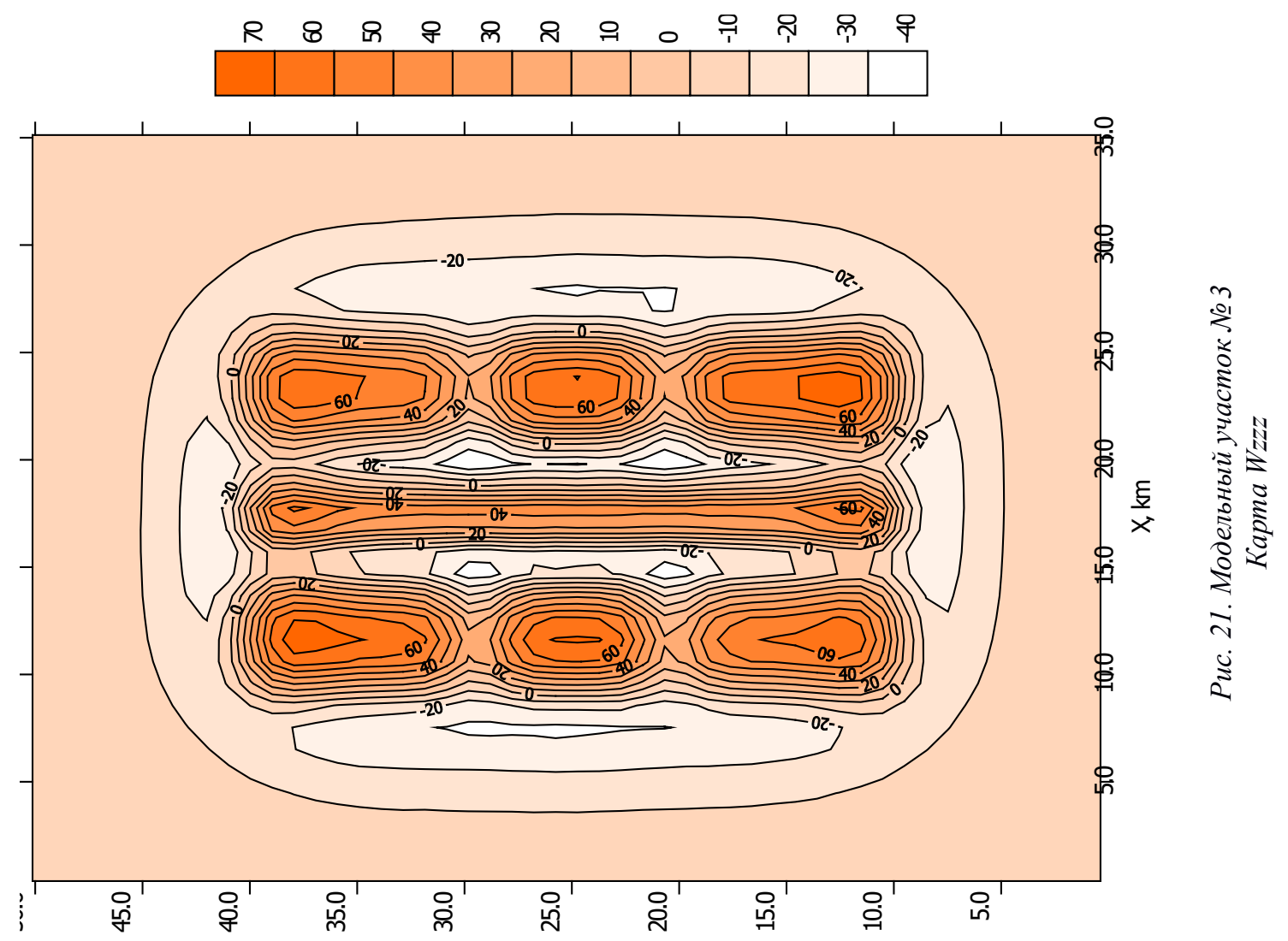




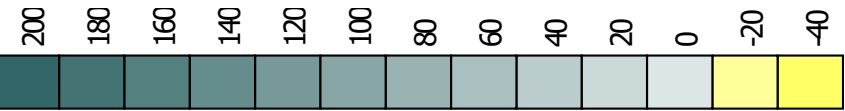

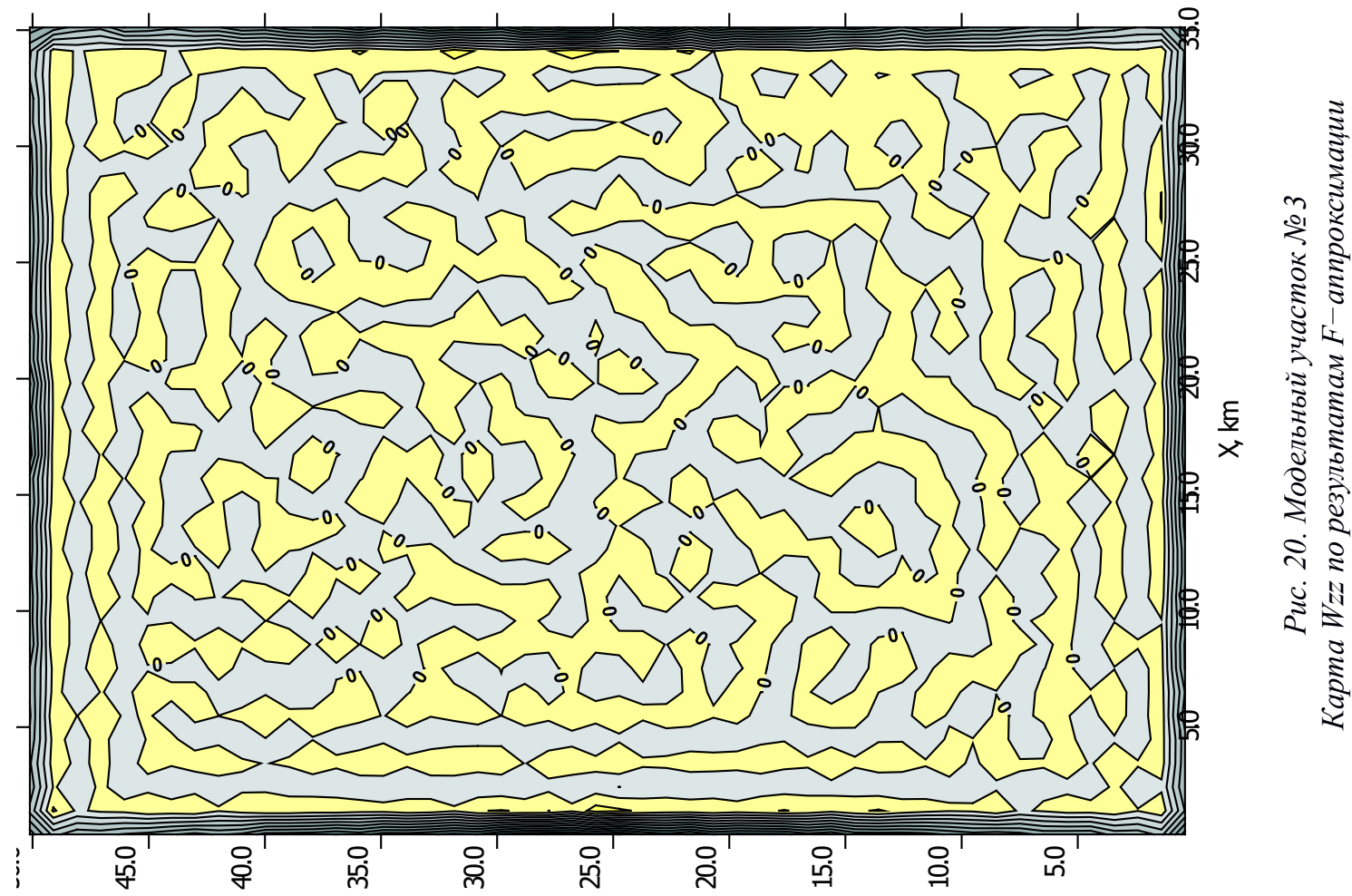

읙 역 그 뜨 늑

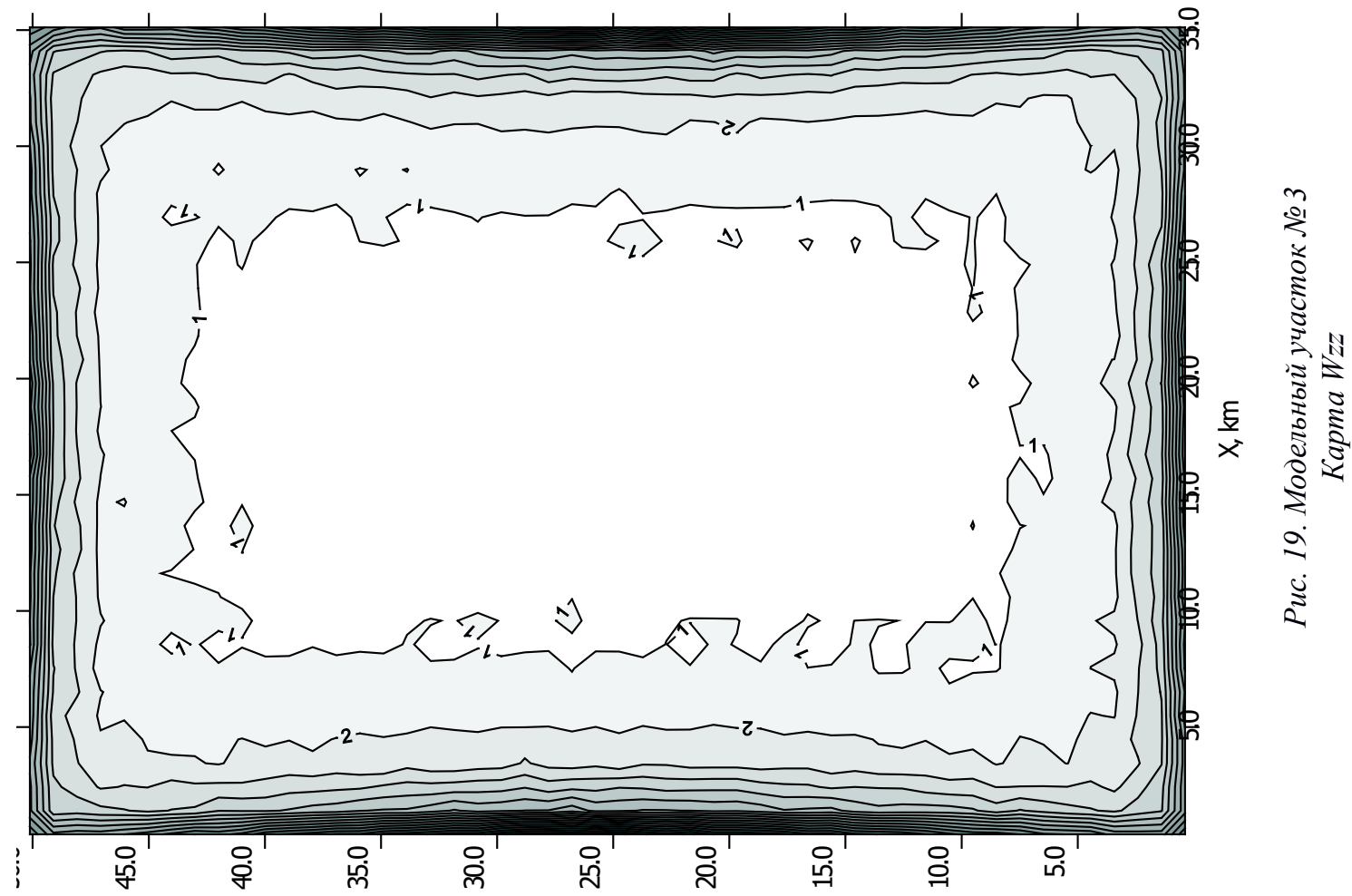




\section{\& \& \& \& \& \& \& \& \& \& \& \& \&}

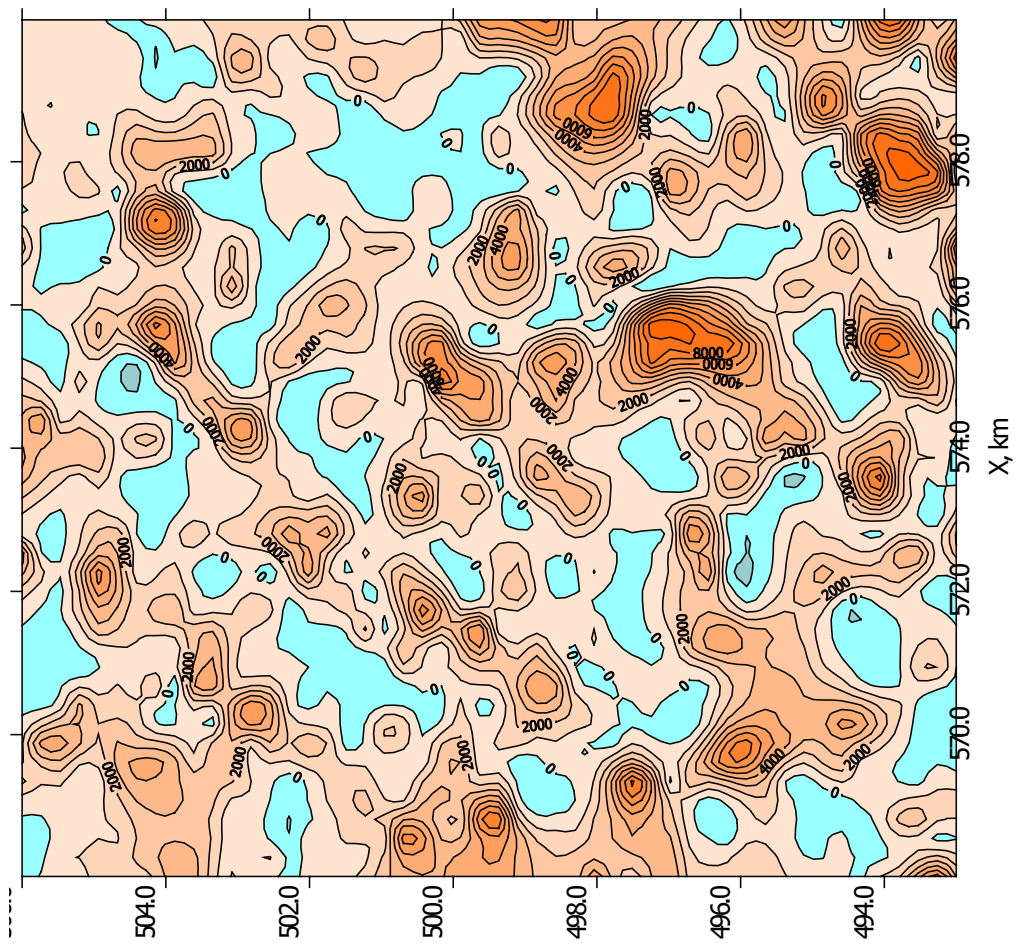

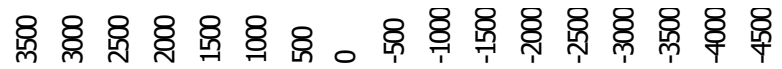

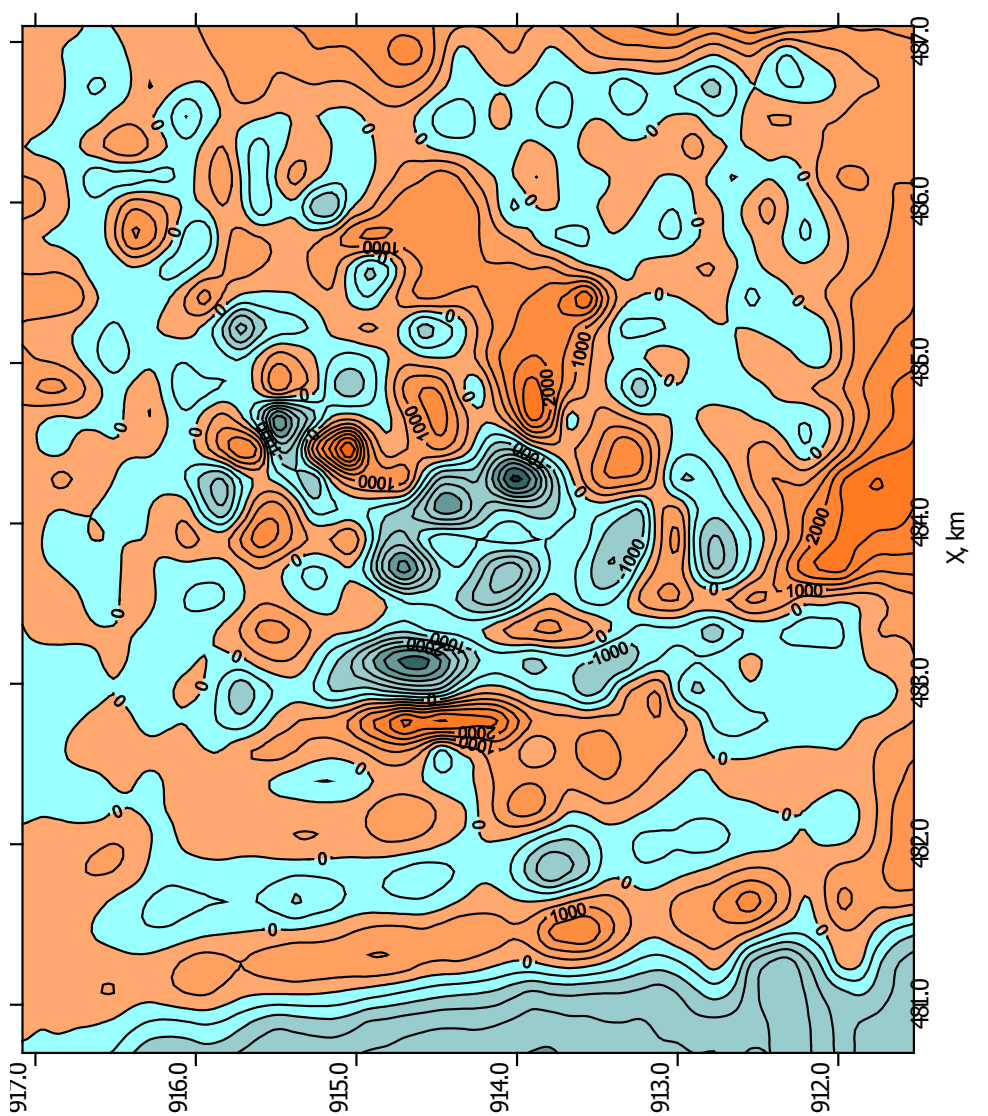

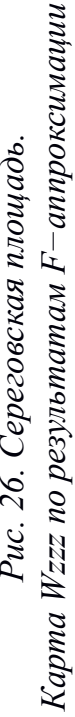


Результаты расчетов производных гравитационного поля на приведенных выше модельных и фактических материалах, а также на ряде других модельных и реальных геолого-гравиметрических данных, позволяют сделать вывод о высокой эффективности вычисления высших производных потенциала силы тяжести на основе F-аппроксимации.

\section{Выводы}

Анализ результатов вычисления высших производных гравитационного потенциала гравитационного поля на основе метода F-аппроксимации, выполненных на модельных и фактических геолого-гравиметрических данных позволяет сделать следующие выводы:

1. Метод $\mathrm{F}$-аппроксимации является высокоэффективным способом вычисление высших производных гравитационного потенциала, обладающий высокой точностью и устойчивостью к ошибкам в исходных данных.

2. Вычисление высших производных гравитационного потенциала на основе метода F-аппроксимации является эффективным инструментом разделения локальных гравитационных аномалий от близко расположенных геологических тел.

3. Карты модуля горизонтального градиента $(W s z)$ и 2-х и 3-х вертикальных производных $(W z z, W z z z)$ потенциала силы тяжести целесообразно использовать для трассирования разрывных нарушений.

4. Для выделения малоамплитудных локальных поднятий осадочного чехла и рифовых тел эффективным является использование карт 2-х и 3-х вертикальных производных ( $W z z, W z z z)$ потенциала силы тяжести.

5. Периферийные участки (около $5 \%$ от всей площади вносят максимальный вклад в погрешность). Погрешность вычисление высших производных гравитационного потенциала без учета точек периферийной зоны значительно ниже погрешности вычисления производных по всей площади.

\section{Литература}

1. Гравиразведка: Справочник геофизика/Под ред. Мудрецовой Е.А. и Веселова К.Е. - М.: Недра, 1990. - 607 с.

2. Керимов И.А. Использование F-аппроксимации при интерпретации гравиметрических данных. І. Методика и результаты опробования на модельных примеpax // Физика Земли, 2003. - № 1. - С. 57-76.

3. Керимов И.А. Использование F-аппроксимации при интерпретации гравиметрических данных. II. Результаты опробования на материалах гравиметрических и магнитометрических съемок // Физика Земли, 2009а. - № 5. - С. 77-93.

4. Керимов И.А. F-аппроксимации рельефа земной поверхности // Физика Земли, 2009б. - № 8. - С. 101-112.

5. Керимов И.А. Метод $\mathrm{F}-$ аппроксимации при решении задач гравиметрии и магнитометрии. - М.: Физматлит, 2011а. - 264 с.

6. Керимов И.А. Теория и компьютерные технологии аналитических аппроксимаций аномальных потенциальных полей // Геология и геофизика Юга России, 2011б. - №2. - С. 50-63.

7. Керимов И.А. Аналитическое продолжение потенциальных полей на основе F-аппроксимации // Геология и геофизика Юга России, 2015. - № 1. - C. 19-32.

8. Керимов И.А., Гайсумов М.Я., Абубакарова Э.А. К вопросу о вычислении высших производных гравитационного потенциала // Вестник Академии наук Чеченской Республики, 2008. - № 2. Т. 1. - С. 53-62. 
9. Маловичко А.К., Тарунина О.Л. Использование высших производных при обработке и интерпретации геофизических наблюдений. - М.: Недра, 1981. - 186 с.

10. Страхов В.Н. Алгоритмы редуцирования и трансформаций аномалий силы тяжести, заданных на физической поверхности Земли // Интерпретация гравитационных и магнитных полей. - Киев: Наукова думка, 1992. - С. 4-81.

11. Страхов В.Н. Три парадигмы в теории и практике интерпретации потенциальных полей (анализ прошлого и прогноз будущего). - М.: ОИФЗ РАН, 1999. - 78 c.

12. Страхов В.Н., Керимов И.А. Аппроксимационные конструкции спектрального анализа (F-аппроксимация) гравиметрических данных // Физика Земли, 2001. - № 12. - C. 3-20.

13. Страхов В.Н., Керимов И.А., Степанова И.Э. Разработка теории и компьютерной технологии построения линейных аналитических аппроксимаций гравитационных и магнитных полей. - М.: ИФЗ РАН, 2009. -254 с.

14. Страхов В.Н., Токарь Л.М. О вычислении высших производных и аналитическом продолжении двухмерных потенциальных полей на основе аппроксимации алгебраическими полиномами // Решение прямой и обратной гравиметрии и магнитометрии (вопросы теории и методики). - М.: ИФЗ, 1985. - С. 4-76.

15. Тарунина О.Л. Структурно-картировочные возможности гравиразведки в комплексе геолого-геофизических исследований. - Пермь: ПГУ, 1993. - 200 с.

DOI: $10.23671 /$ VNC.2016.3.20831

\title{
CALCULATION OF HIGHER DERIVATIVES OF THE GRAVITATIONAL POTENTIAL ON THE BASIS OF F-APPROXIMATION
}

\author{
() 2016 I.A. Kerimov, Dr. Sc. (Phys.-Math.), Prof.
}

The Schmidt Institute of Physics of the Earth of the Russian Academy of Sciences,

Russia, 123995 Moscow, B. Gruzinskaya st., 10-1, e-mail: kerimov@ifz.ru

This article considers an approximate method of the gravitational field transformation (the determination of different components of the gravity potential). The method developed by the author and computer technologies of the F-approximation permit the calculation of horizontal and vertical derivatives of the gravitational potential for basic data defined both on a regular and on an irregular grid. The method is tested on model and actual gravimetric data.

Keywords: approximation, higher derivatives, anomaly, gravitational field, transformation. 